\title{
Context-aware negotiation, reputation and priority traffic light management protocols for VANET-based smart cities
}

\author{
Nabila Bermad, Salah Zemmoudj, and Mawloud Omar \\ \{nabila.bermad, salah.zemmoudj\}@univ-bejaia.dz,mawloud.omar@gmail.com

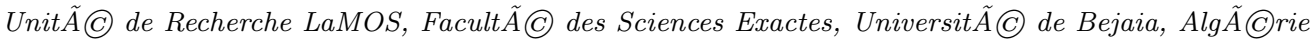

\begin{abstract}
The traditional transportation system is based on a fixed-timed strategy to control the traffic congestion on urban roads. However, the increase of vehicle density in a smart city implies the variation and the conflict on the demand pattern of the drivers. The smart city development requires to create an efficient control plan for an intelligent traffic management system. In this paper, we aim to reduce the congestion at signalized intersections, and satisfy the needs of drivers according to their degree of displacement urgency. We use two optimization methods, namely the synchronization and the Genetic Algorithm (GA), where we develop three scheduling protocols. The first is the intelligent context-aware negotiation protocol (ICANP). This protocol allows the negotiating vehicles to cross the intersection. It enables each traffic light at the signalized intersection to negotiate the green time assigned to its phase. ICANP uses GA to optimize the crossing time in order to minimize the total waiting time of negotiating vehicles. Moreover, we introduce a negotiation protocol based on reputation (NPBR), which minimises the congestion effect from incoming dishonest drivers. Finally, we propose an intelligent context-aware priority protocol (ICAPP), that considers the existence of priority vehicles. Upon arrival of at least one priority vehicle at the signalized intersection, ICAPP interrupts the green time of negotiating vehicles. A series of simulation showed that the proposed protocols reduce the total waiting time and the emissions of $\mathrm{CO}_{2}$ of vehicles at signalized intersection, in comparison with circular, ITLC and CATLS scheduling algorithms. Furthermore, formal complexity analysis and performance evaluation show the effectivity of our protocols.
\end{abstract}

Keywords: Smart City, VANET, Traffic Light, Negotiation, Reputation, Priority vehicle.

\section{Introduction}

The smart city paradigm is the result of integrating information and communication technologies, which improves the functioning of different services in cities. Transport service is one of the main sectors that make life easier for citizens. With the increasing use of vehicles, the problem of road congestion becomes the most penalizing phenomenon of road users, especially at the signalized intersections. Implementing the vision of a smart vehicular system has been performed facing this issue. In this view, VANET is used to collect real-time information about traffic flow at a road intersection. Afterward, data acquired serves as input to an intelligent traffic light scheduling algorithm to generate the traffic signal timing.

By nature, many drivers want to reach their destination as soon as possible. Arriving earlier can decrease the travel time of vehicles on the road, which minimizes the emissions of $\mathrm{CO}_{2}$ and conserve the green environment in the city. Several researchers have developed an intelligent, and effective strategy to schedule the competing flows of traffic at signalized road intersection [1, 2], 3], 4] [5], [6]. These strategies are aimed at either minimizing the average 
delay per vehicle or decreasing the queue length of vehicles at intersections. However, some of the existing gaps in the previously proposed solutions are: (1) Did not integrate the security requirements such as authentication against the malicious behavior of drivers. 2) Except [2], the previous proposals did not consider the dynamic driver's context (displacement urgency and final destination) to adapt the timing signal at intersection. In this paper, we propose three synchronization protocols to optimize the traffic signal control at signalized intersection. The most critical issues to be resolved are: How to define the intersection control methods in a connected vehicle environment, taken into account the vehicle priorities, and how to judge the vehicle's reputation and take appropriate decisions. Unlike the previous cited works, the underlying optimization problem in our proposal aims to reduce the $\mathrm{CO}_{2}$ emissions. In the context of our work, we proceed by classifying vehicles between priority, concurrent and regular. A priority vehicle represents a trusted vehicle with very high travel urgency. It can cross the intersection without negotiation. A concurrent vehicle is a vehicle with critical travel urgency. It negotiates the passage at the intersection. The traffic light allows it to cross the intersection after the evaluation of its reputation value. A regular vehicle is a vehicle with no urgency. Each traffic light $L_{i}(i=1 \cdots n)$ supervises a set of vehicles, where $n$ refers to the number of traffic lights at the intersection. $L_{i}$ synchronizes dynamically the green time with the other traffic lights. It negotiates the tipping of the passages according to the priorities of its vehicles. The traffic light which supervises the highest density of vehicles allows them to proceed first. However, there are dishonest vehicles, as they can cheat in their urgency, which increases the congestion at the signalized intersection. Accordingly, the traffic light exploits the obtained reputation by using VANET to minimize their effects.

The synchronization strategy between traffic lights maximizes the number of vehicles, which cross the intersection at a given moment. However, a fixed quantum $\mathrm{Q}_{i}$ increases the congestion. For that, the traffic light urges the vehicles to arrive at their destinations in a minimal time, and then, the longest waiting time must be minimized. Many researchers used the meta-heuristic approaches to treat the traffic congestion, and to change the real time phase duration depending on the traffic flow of each traffic light. In [7, 8, Garcia Nieto et al. proposes a new particle swarm optimizer (PSO), which is capable of obtaining efficient cycle programs for realistic urban scenarios. In this new approach, the initialization method, the solution encoding, the fitness function, and the velocity calculation have been adapted to deal with optimal traffic light cycle program. Moreover, they focus on the improvement of the traffic flow with the global purpose of reducing contaminant emissions $\left(\mathrm{CO}_{2}\right.$ and $\left.\mathrm{NO}_{x}\right)$ and fuel consumption in the analyzed areas. In [9], Chin et al. proposes a traffic signal timing management (TSTM) system, which comprises of genetic algorithm based signal optimization with the nature characteristic of genetic algorithm to search the most optimum solution by evolutions. This approach allows signal timing parameters such as offset, cycle time, green split and phase sequence to be optimized with the objective of minimum delay and better traffic fluency. In [10, Teo et al. proposes traffic flow control optimization in traffic light system. They study the effect of queue length, green time, cycle time and amber time in the traffic system. Furthermore, the genetic algorithm is used in the traffic flow control to optimize the traffic flow in the intersection. In [11, Singh et al, proposes a real-time traffic signal control strategy using genetic algorithm to provide near optimal traffic performance for intersections. They develop emulator for representation of traffic conditions at an isolated intersection. Factors considered for genetic optimization are: (a) weights allotted to each road, (b) fixed maximum and minimum green timings, and (c) fixed cycle timings and total stop timings at each incoming lane. The authors in [12] introduce a multi-objective evolutionary algorithm NSGA - II [13] to obtain a relatively better signal timing plan for oversaturated intersection. Throughput maximum and average queue ratio minimum are selected as the optimization objectives of the traffic signal control under oversaturated condition. NSGA - II algorithm was tested under various kinds of phases and traffic conditions. However, the proposed algorithm is difficult to get feasible solution if the large gene number is selected because of the computational complexity. Recently, the authors in [14] present a fog computing archi- 
tecture network (FOCAN). This architecture shows how smart city components and services can communicate with each other and fog computing. To this end, the smart city is comprised of several heterogeneous components, such as smart mobility, a smart grid, smart surveillance, and so on. The FOCAN is a computation and communication-efficient structure and scalable routing algorithm. It minimizes the average power consumption of FNs, when it serves numerous requests coming from various devices such as vehicle, traffic light, etc. The authors of 24 have continued in the same direction by proposing a fog-based security framework for intelligent traffic light control management. It ensures several services, such as confidentiality, integrity, and authentication. It consists of three main components: (1) Vehicle, which is the central entity needing to be assisted in travel plans; (2) Road Side Unit (RSU), responsible for broadcasting encrypted messages and certificate in the monitored region; and (3) Department of Motor Vehicles (DMV), which is a trusted government agency, responsible for installing of RSUs, and vehicles registration.

The main objective of this paper is to minimize traffic congestion at signalized intersections, taking into account the dynamic context of drivers. We divide this problem into two complementary phases: the first is to maximize the number of vehicles reaching their destinations, while the second focuses on the dynamism of $Q_{i}$, which offers optimized crossing time to minimize longer stay of each vehicle. Here, we propose three synchronization protocols. The intelligent context-aware negotiation protocol (ICANP) minimizes the congestion on single $\mathrm{n}$ way intersection. ICANP satisfies the drivers requirements in real time based on their degree of displacement urgency. ICANP adapts dynamically the crossing time assigned at the phase of each traffic light. We use the genetic algorithm to optimize the crossing time of negotiating vehicles in order to decrease their total waiting time at signalized intersection road. The genetic algorithm takes the effective density of each traffic light, and generate the negotiating vehicles groups of different size. ICANP interrupts the green time assigned at the phases of regular vehicles upon the arrival of negotiating vehicles to pass them fast at signalized intersection. Secondly, we also introduce the negotiation protocol based on reputation (NPBR). This protocol aims to minimize the congestion effect from incoming dishonest vehicles. It uses the reputation of drivers to calculate the volume of dishonest vehicles. In NPBR, priority is given to a group of negotiating vehicles, that has a minimum volume of dishonest vehicles. It uses the genetic algorithm to optimize the crossing time. Hence, minimize the total waiting time at signalized intersection. Thirdly, we propose an intelligent context-aware priority protocol (ICAPP). The latter considers the priority vehicles that have heaviest reputation. The ICAPP allows them to cross the intersection without negotiation. In addition, ICAPP privileges the priority vehicle group, which has a higher average reputation than other priority groups of the same density. On arrival of at least one priority vehicle at the signalized intersection, the ICAPP interrupts the green time of negotiating vehicle groups.

In the proposed control system based on the connected vehicle environment, the drivers do not assign themselves roles with priority. However, the traffic light is the one that attributes the role to the drivers taking into account their travel urgency. In the case of concurrent vehicles, upon receiving the vehicle to light negotiation request, the traffic light uses the ICANP to maximize the number of negotiating vehicles reaching their destinations. Then, it calculates the vehicle crossing time based on genetic algorithm (GA) to minimize longer stay of each negotiating vehicle and makes decisions that avoid conflicts. If a vehicle approaching intersection is priority vehicle (e.g. Police car). The traffic light uses the ICAPP to allow the priority vehicle to cross the intersection without negotiation. Besides, in order to detect dishonest drivers, the traffic light introduces negotiation protocol based on reputation (NPBR) to minimize the effect of dishonest vehicles on congestion. Finally, for the regular vehicle, the traffic light allowed them the passage according to circular protocol.

The remaining of this paper is structured as follows. In section 2, we present the related work. In Section 3, we present a detailed description of the proposed approach. In Section 4 . we present the simulation result of our approach. Finally, we conclude the paper in Section 5 . 


\section{Related Work}

Intelligent transportation systems (ITS) use communication technology to connect the vehicles for more secure, safer, and highly mobile transportation in urban environments. However, the rapid increase in the vehicles density causes congestion that attracts great attention due to the network infrastructure degradation, especially at the signalized intersection. In this context, various researches have been performed in the recent years.

Soylemezgiller et al. [15] proposed a radically dissimilar road pricing scheme to avoid and reduce the traffic clogging in metropolises. The road pricing system over the entire city has been recommended. This system can manage the traffic flow in the entire traffic network of the area. Also, the road costs are adjusted dynamically on the basis of instantaneous traffic densities of every road in the smart city in order to avoid the traffic congestion.

Besides, Younes et al. 1] introduced an intelligent traffic light controlling (ITLC) algorithm. ITLC used VANET to gather the real-time traffic characteristics of all competing traffic flow at each signalized intersection. ITLC schedules all the traffic flows. It starts with the platoon that has the heaviest density. Moreover, it integrates an arterial traffic light ( $A \Pi \mathrm{L}$ ) controlling algorithm. With $A T L$, ITL installed at each road intersection coordinate to generate an efficient traffic schedule for the entire road network.

In order to consider the presence of emergency vehicles on competing flows, Younes et al. 2. presented a context-aware traffic light scheduling (CATLS) algorithm. CATLS considered the traffic characteristics of traffic flows to schedule the phases of each traffic light. The green phase of any traffic flow can be interrupted to enable the fast proceeding of appearing emergency vehicles. In the case where more than one emergency vehicle may arrive the road intersection at the same time, CATLS scheduled the sequential phases of traffic light, according to the prioritie of each vehicle to cross the intersection. If the priorities of the un-conflicted flows were equivalent, the one with highest traffic density was selected.

An adaptive traffic light controlling algorithm was proposed in [3. It used the per-vehicle real time position and speed data to perform traffic scheduling at an isolated traffic intersection. The authors formulated the vehicular traffic signal control as a job scheduling problem on processors, where the jobs correspond to the platoons of vehicles. Under the assumption that all the jobs are of equal size. An on-line algorithm (OAF) was developed to reduce the waiting delay time of traveling vehicles. This algorithm schedules the most efficient sequence of phases of each traffic light cycle.

In the case of an urban arterial road, Tomescu et al. 16 investigated the problem of adaptive traffic light control. They considered real-time traffic information taking into account drivers behavior, traffic control and environmental factors. The arterial network system (ARTSYS) was proposed for the arterial street scenario. In this system, every intersection is controlled by its own traffic volume, vehicle type and neighboring intersections cooperative recommendations. These parameters are taken into consideration by an adaptive traffic light control strategy for adjusting the offset time.

Fogue et al. [17 developed eNOTIFY system designed for automatic accident detection. eNOTIFY sends the message to the emergencies coordination center and assistance of road accidents via the VANET network. This system focuses on improving post collision care with a fast management of the available emergency resources, which increases the chances of recovery and survival for those injured in traffic accidents

The work in [4, 18 is a new scheme, which consists of a smart city framework for intelligent traffic system using VANET. The proposed system is based on intelligent traffic lights that are set up at crossroads of a city. The functions of these ITLs are the collection of traffic information, such as traffic density of passing vehicles, updating congestion information and also reporting these traffic statistics to individual vehicles, which are close to ITLs.

Another ITS system [19] that presented a direct relation between the emissions of a given car and its speed or acceleration. It aimed to periodically guide the drivers through intersections 
equipped with ITLs. It recommended optimal speeds to reduce the number of vain accelerations to catch green lights, and the number of stop-starts due to red lights.

In [20, Gupta et al. proposed methods for intelligent control of traffic lights in order to manage and resolve the road congestion incidents. An optimal traffic light sequence has been obtained for a single intersection using the hop-field neural network (HNN). The optimal green time for the traffic lights has been obtained using genetic algorithm (GA), which maximizes the traffic flow, because it has found that the flow rate increased with the increase in green times.

Another protocol to control congestion was found by Santamaria et al.21], which developed a new smart traffic management protocol (STMP). STMP reduced the level of traffic in the city using V2V and V2I communications. Moreover, the real-time control of the way helps to detect blocked roads in the city, and traffic jams in the shortest time. STMP made possible to control the congestion initially by better traffic management.

Bravo et al. 22] developed a decision support system that helps traffic managers to generate an optimal traffic light plane (TLPs) for actual cities. This system took the real traffic conditions. Furthermore, it computed optimal traffic lights plans using bio-inspired techniques and microsimulation.

Wen et al. 23 provided a framework for a dynamic, automatic traffic light control expert system combined with a simulation mode. It consisted of six sub-models coded in Arena to help analyze the traffic problem. Each sub-model represents a road that has three intersections. It adopted inter-arrival time and inter-departure time. It simulated the arrival and leaving a number of cars on roads. The expert system combined with a traffic light control simulation model reduces the average waiting time of cars at every intersection.

The most of these studies have focused on different aspects of the congestion management. However, a common limitation to all is that they have considered just reducing the waiting time of vehicles. They do not take into account all possible scenarios, that minimize both congestion at intersections and satisfy the dynamic context of the drivers. Also, none of them considered the all types of vehicles, according to their degree of urgency. They do not study the effect of drivers reputation on congestion. We summarize in Table 1, the strengths and weaknesses with existing solutions extensively highlighted in Section 2 In contrast, our proposal is a general framework that deals with all scenarios of congestion whatever the type of vehicle, and satisfies the need of drivers. Moreover, we provide a reputation model, which allows the traffic light to evaluate and manage the reputations of its vehicles and act accordingly.

\begin{tabular}{|c|c|c|}
\hline Protocol & Strengths & Weaknesses \\
\hline 1] & $\begin{array}{l}\text { - Reducing the queuing delay of each } \\
\text { vehicle. } \\
\text { - Increasing the throughput of the in- } \\
\text { tersection. }\end{array}$ & $\begin{array}{l}\text { - The priorities between vehicles in the } \\
\text { same traveling platoons, or different } \\
\text { platoons with the same density are ne- } \\
\text { glected in the traffic light scheduling } \\
\text { decision. } \\
\text { - Not context aware (degree of urgency). } \\
\text { - The reputation of drivers is neglected. }\end{array}$ \\
\hline 2 & $\begin{array}{l}\text { Eliminating the delay of emergency ve- } \\
\text { hicles at the signalized intersection }\end{array}$ & $\begin{array}{l}\text { - Increasing the delay of the other trav- } \\
\text { eling vehicles. } \\
\text { - Not deal with all possible scenarios } \\
\text { with other types of vehicles (negotiat- } \\
\text { ing, regular.) } \\
\text { - The reputation of drivers is neglected. }\end{array}$ \\
\hline
\end{tabular}




\begin{tabular}{|c|c|c|}
\hline 3 & $\begin{array}{l}\text { Minimizing the average delay per vehi- } \\
\text { cle }\end{array}$ & $\begin{array}{l}\text { - Not context aware } \\
\text { - The reputation of drivers is neglected }\end{array}$ \\
\hline 15 & $\begin{array}{l}\text { Avoiding and reducing the traffic clog- } \\
\text { ging in metropolises. }\end{array}$ & $\begin{array}{l}\text { If the suggested road is long, then, it in- } \\
\text { crease the traveling delay of urgency ve- } \\
\text { hicles to reach their destination. }\end{array}$ \\
\hline 16 & $\begin{array}{l}\text { Reducing the stop number and the de- } \\
\text { lay of each vehicle }\end{array}$ & $\begin{array}{l}\text { - Not context aware. } \\
\text { - The reputation of drivers is neglected. }\end{array}$ \\
\hline 17 & $\begin{array}{l}\text { - Improving post collision care using } \\
\text { VANET network. } \\
\text { - Automatic accident detection. }\end{array}$ & $\begin{array}{l}\text { The traffic light scheduling problem is not } \\
\text { addressed. }\end{array}$ \\
\hline 4, 18 & $\begin{array}{l}\text { Decreasing the waiting time of vehicles } \\
\text { at crossroads and helping to prevent } \\
\text { collisions. }\end{array}$ & $\begin{array}{l}\text { - Not considering the reputation of } \\
\text { drivers when a congestion free path is } \\
\text { calculated. } \\
\text { - Not context aware. }\end{array}$ \\
\hline 19 & Reducing vehicle emissions & $\begin{array}{l}\text { - Not context aware. } \\
\text { - Requiring traffic light scheduling algo- } \\
\text { rithm. }\end{array}$ \\
\hline 20 & Maximizing throughput & $\begin{array}{l}\text { - Not context aware. } \\
\text { - The reputation of drivers is neglected. }\end{array}$ \\
\hline 21 & $\begin{array}{l}\text { - Reducing the average delay } \\
\text { - Reducing the } \mathrm{CO}_{2} \text { emission. }\end{array}$ & $\begin{array}{l}\text { - Not context aware. } \\
\text { - The reputation of drivers is neglected. }\end{array}$ \\
\hline 22 & $\begin{array}{l}\text { - Minimizing energy consumption. } \\
\text { - Reducing the congestion. } \\
\text { - Improving pedestrian safety. } \\
\text { - Minimizing pollutant emission. }\end{array}$ & $\begin{array}{l}\text { - Not context aware. } \\
\text { - The reputation of drivers is neglected. } \\
\text { - Not scale to large urban area. }\end{array}$ \\
\hline 23 & $\begin{array}{l}\text { Reducing the average waiting time of } \\
\text { cars at every intersection }\end{array}$ & $\begin{array}{l}\text { - Not context aware. } \\
\text { - The reputation of drivers is neglected. }\end{array}$ \\
\hline Current work & $\begin{array}{l}\text { - Context aware. } \\
\text { - The reputation of drivers is consider- } \\
\text { ing when crossing at the intersection. } \\
\text { - Maximizing the number of vehicles } \\
\text { reaching their destinations. } \\
\text { - Minimizing the total waiting time of } \\
\text { vehicles at signalized intersection. } \\
\text { - Optimizing crossing time of vehicles. } \\
\text { - Deal all possible scenarios with dif- } \\
\text { ferent category of vehicles (priority, } \\
\text { negotiating, and regular). } \\
\text { - Distributed protocols. }\end{array}$ & - \\
\hline
\end{tabular}


Table 1: Strengths and weaknesses of the related works

\section{The proposed approach}

In this section, we present the network model, and then the detailed description of the proposed approach. Table 2 summarizes the notations used in the proposed approach.

\subsection{Network Model}

A connected VANET to smart city infrastructure can have access to more information via the Internet. This infrastructure represents the traffic lights at intersections. It is a regulatory measure of traffic between the road users. The traffic lights usually switch between three basic colors, namely the red light to mark the absolute stop of vehicles, the green light to allow the passage of vehicles, the orange light to signal the passage of the green light to red light, and vice versa. In each intersection, the vehicles send the negotiation request to traffic light in order to cross the signalized intersection. Next, a set of traffic lights synchronize with each other to negotiate the green time of vehicles group, that supervises them.

In the context of our work, we are interested in a single intersection n-way connected to traffic lights infrastructure of the smart city. In Figure 1, we illustrate an example of four-leg intersection with four input way and four output way of traffic flow. The considered network model has the following entities:

a) Vehicles: The vehicles communicate with each other. They send warning messages based on V2V communication and with a traffic light. The latter supervises the vehicles by exchanging their negotiation requests to cross the signalized intersection. Each vehicle has a unique identifier VID. It is equipped with a set of sensors, which measure its speed, position and direction. A vehicle is controlled by its driver, who personalizes initially two informations: (a) The destination adresse is indicated on GPS of the vehicle. (b) The urgency degree that represents a combination of several information, such as the required arrival time, travel journey time.

b) Traffic light: In each intersection, a set of traffic lights $L_{i}(i=1 . . n)$ exchanges control messages to synchronize and switch the passage quantum time $Q_{i}$. Each traffic light supervises a set of vehicle categories in the case of emergency, urgent by negotiation and regular. We assume that traffic light is responsible for splitting the incoming traffic flow into groups, moving together of different density with presence or not of dishonest vehicles. In addition, each traffic light $L_{i}$ is in the wireless communication range of vehicles. It also communicates with other adjacent traffic lights of its neighboring intersections to get information about the driver behavior via their traffic light managers. Each traffic light $L_{i}$ contains dashboard, that allows to display the recommended speed for the negotiating vehicle groups.

c) Trust City Manager (TCM): It is a trusted entity responsible for monitoring the behavior of drivers in the city. It can register the reputation value of historical interaction for each vehicle in VANET. In order to avoid the congestion at the signalized intersections, TCMsends recommendations to traffic lights via the messages trust city manager to light.

\subsection{Exchanged messages}

We classify the messages into three types: 


\begin{tabular}{|c|c|}
\hline Variable Notation & Description \\
\hline $\mathrm{L}_{\mathrm{i}}$ & Traffic light $i$ \\
\hline $\mathrm{Q}_{\mathrm{i}}$ & Quantum time of $\mathrm{L}_{i}$ \\
\hline$v_{j}$ & Vehicle $j$ \\
\hline $\mathrm{n}$ & Number of traffic lights \\
\hline $\mathrm{T}_{i}^{\mathrm{a}}\left(v_{j}\right)$ & Arrival time of $v_{j}$ \\
\hline$d_{i}^{r}$ & Real density of negotiating vehicles \\
\hline$d_{i}^{p}$ & Real density of priority vehicles \\
\hline$C_{i}$ & Local clock of $L_{i}$ \\
\hline$\triangle \mathrm{t}$ & Time of orange light \\
\hline $\mathrm{C}_{\mathrm{i}}^{\mathrm{t}}$ & Crossing time of vehicles supervised by $\mathrm{L}_{i}$ \\
\hline$P_{i}^{t}$ & Predefined time of vehicles supervised by $L_{i}$ \\
\hline$S_{i}^{t}$ & Stop time of vehicles supervised by $L_{i}$ \\
\hline$v_{l}$ & Last vehicle \\
\hline $\mathrm{D}_{\mathrm{i}}^{\mathrm{e}}\left(v_{\mathrm{l}}\right)$ & Effective distance between the $v_{l}$ in traffic flow authorized to pass and $\mathrm{L}_{i}$ \\
\hline$S^{r}$ & Recommended speed \\
\hline$d_{i}^{e}$ & Effective density of vehicles authorized to pass \\
\hline$t_{i}\left(v_{j}\right)$ & Delay time to start and to reach at $S^{r}$ for $v_{j}$ supervised by $L_{i}$ \\
\hline $\mathrm{T}^{\mathrm{c}}$ & Time cycle \\
\hline D & Real distance \\
\hline $\lg$ & Total length of vehicles \\
\hline h & Total safety distance \\
\hline$T^{w}$ & Total waiting time \\
\hline$t_{i}^{w}\left(v_{j}\right)$ & Waiting time spent by a $v_{j}$ at each $L_{i}$ \\
\hline $\mathrm{T}_{i}^{\mathrm{g}}$ & Start time of green light at each $L_{i}$ \\
\hline$C_{i}^{t}\left(v_{j}\right)$ & Crossing time of each $v_{j}$ supervised by $\mathrm{L}_{i}$ \\
\hline $\mathrm{T}_{i}^{\mathrm{g}}$ & Start time of green light \\
\hline$C_{i}^{t}\left(v_{j}\right)$ & Crossing time of each $v_{j}$ \\
\hline$D_{i}^{e}\left(v_{j}\right)$ & Effective distance between the $v_{j}$ and the $L_{i}$ \\
\hline$P_{i}$ & Represents the existence ( 1 or 0 ) of priority vehicles supervised by $L_{i}$ \\
\hline$R_{i}^{j}$ & Reputation value of each $v_{j}$ supervised by $L_{i}$ \\
\hline$V_{i}^{D}$ & Volume of dishonest vehicles supervised by $L_{i}$ \\
\hline$t_{i}^{w}\left(v_{j}^{\mathrm{H}}\right)$ & Waiting time spent by each honest $v_{j}$ supervised by $L_{i}$ \\
\hline$C_{i}^{t}\left(v_{k}^{D}\right)$ & Crossing time of each dishonest vehicle $k$ supervised by $L_{i}$ \\
\hline$d_{i}^{(r, H)}$ & Real density of honest vehicle supervised by $L_{i}$ \\
\hline$d_{i}^{(r, p)}$ & Real density of priority vehicle supervised by $L_{i}$ \\
\hline$R_{i}^{A}$ & Average reputation of vehicles supervised by $L_{i}$ \\
\hline
\end{tabular}

Table 2: Notations 


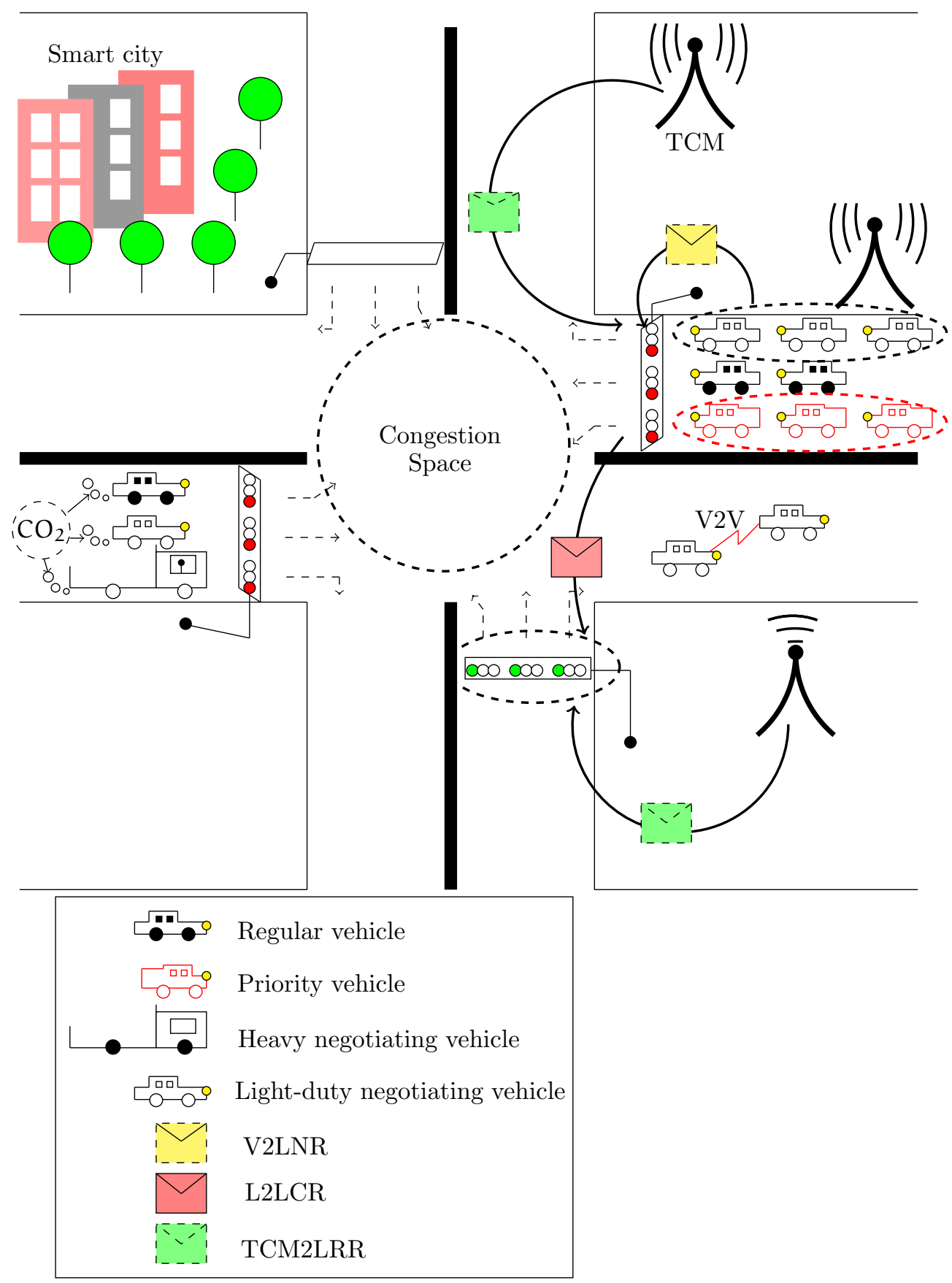

Figure 1: Network model and assumptions 
a) Vehicle to Light Negotiation Request (V2LNR): The vehicles arriving at the signalized intersection can request the passage. They send to the traffic lights a V2LNR control message $\left\langle\mathrm{VID}_{i}^{j}, \mathrm{RD}^{j}, \mathrm{U}^{j}, \mathrm{DS}^{j}, \mathrm{DID}^{j}\right\rangle$, where $\operatorname{VID}_{i}^{j}$ is the identifier of the vehicle $j$ supervised by the traffic light $L_{i}, R D^{j}$ is the requested direction of intersection by the driver to reach his final destination, $\mathrm{U}^{j}$ is the $v_{j}$ displacement urgency, $\mathrm{DS}^{j}$ is the $v_{j}$ destination address, and DID ${ }^{j}$ is the driver identifier of $v_{j}$. In the context of our work, we define three classes of vehicles, according to their travel urgency: (1) The priority vehicles represent a very high urgency without negotiation (e.g., police, ambulance, etc.), where the traffic lights prioritize them in the intersection; (2) concurrent vehicles under negotiation (e.g., service vehicles, taxi, vehicles with critical urgency, etc.), which negotiate the passage at the intersection; and (3) regular vehicles with low priority.

b) Light to Light Control Request (L2LCR): When receiving V2LNR messages, the traffic light $\mathrm{L}_{i}$ stores the arrival time $\mathrm{T}_{i}^{\mathrm{a}}\left(v_{j}\right)$ of each $v_{j}$. It synchronizes with the others traffic lights to negotiate the green time via a message $L 2 L C R\left\langle d_{i}^{r}, d_{i}^{p}, C_{i}\right\rangle$, where $d_{i}^{r}$ and $d_{i}^{p}$ represents, respectively, the real density of negotiating and priority vehicles supervised by the $L_{i}$, and $C_{i}$ represents the local clock of $L_{i}$.

c) Trust City Manager to Light Recommended Request (TCM2LRR): TCM sends a recommendation of each vehicle to requested traffic light, that represents the historical reputation of the vehicle in VANET network.

\subsection{Local and global synchronization protocols}

The local synchronization protocol, illustrated in Algorithm 1, is designed to locally manage the messages exchanging between the game lights of each $L_{i}$, that alternately passes from green to red and from red to green, taking a time of some seconds $\Delta t$ for the orange light to signal the switching of colors red and green. The $Q_{i}$ of green light is assigned to each phase of $L_{i}$. It represents the crossing time $C_{i}^{t}$ for negotiation case or the predefined time $P_{i}^{t}$ for circular case. The value of $Q_{i}$ is estimated by,

$$
Q_{i}= \begin{cases}P_{i}^{t}, & \text { circular case } \\ C_{i}^{t}, & \text { otherwise }\end{cases}
$$

however, when the stop time $S_{i}^{t}$ of the signal red is triggered, the vehicles are not allowed to cross the intersection. 


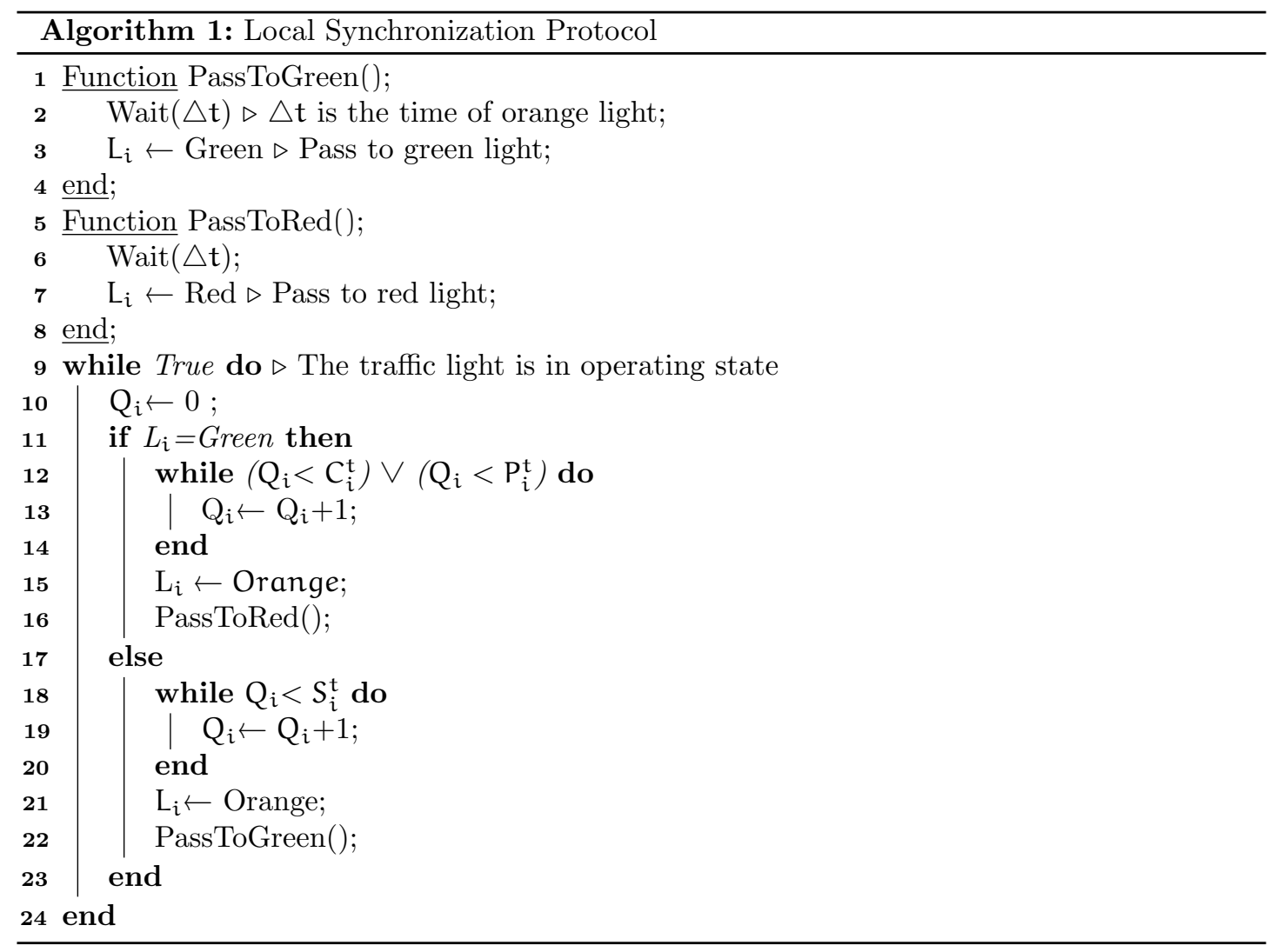

Unlike the local synchronization protocol that controls the switching of game lights for each traffic light, the global synchronization protocols are designed to synchronize between different traffic lights by exchanging messages. They allow the concurrents traffic flows to cross the intersection. The circular synchronization protocol, illustrated in Algorithm 2, is an integral part of these protocols. It cyclically assigns the green light to each traffic light $L_{i}$, with a predefined time $P_{i}^{t}$ in order to allow the vehicles to cross the signalized intersection. This protocol is adequate to manage the signal timing phase of regular vehicles. But, it does not minimize the waiting time of urgent vehicles at the signalized intersection. In our work, we propose two synchronization cases, namely negotiation and priority. The negotiation case assigns dynamically the green light to each traffic light, according to the displacement urgency of the drivers. However, the priority case allows the priority vehicles to cross the signalized intersection without negotiation. These cases aim to minimize the congestion, and satisfy the drivers context by improving the waiting time of travelling vehicles.

\subsubsection{Negotiation Case}

Depending on the drivers context of negotiating vehicles, we introduce an intelligent contextaware negotiation protocol (ICANP). The ICANP considers the urgency of negotiating vehicles to allow the traffic lights to negotiate the crossing time of their concurrent traffic flow. This protocol aims to minimize the waiting time of negotiating vehicles at the signalized road intersection. It optimizes the $C_{i}^{t}$ for circular, negotiation and priority protocols mentioned above. The $C_{i}^{t}$ is calculated such as, 


$$
C_{i}^{t}=\frac{D_{i}^{e}\left(v_{l}\right)}{S^{r}}+\sum_{j=1}^{d_{i}^{e}} t_{i}\left(v_{j}\right)
$$

where $D_{i}^{e}\left(v_{l}\right)$ is the effective distance between the last vehicle $v_{l}$ in traffic flow authorized to pass and the $L_{i}$ that supervised it, $S^{r}$ is the recommended average traffic speed at signalized intersection, $d_{i}^{e}$ is the effective density of vehicles authorized to pass, and $t_{i}\left(v_{j}\right)$ is the delay time to start and to reach at $S^{r}$ for each $v_{j}$ supervised by $L_{i}$.

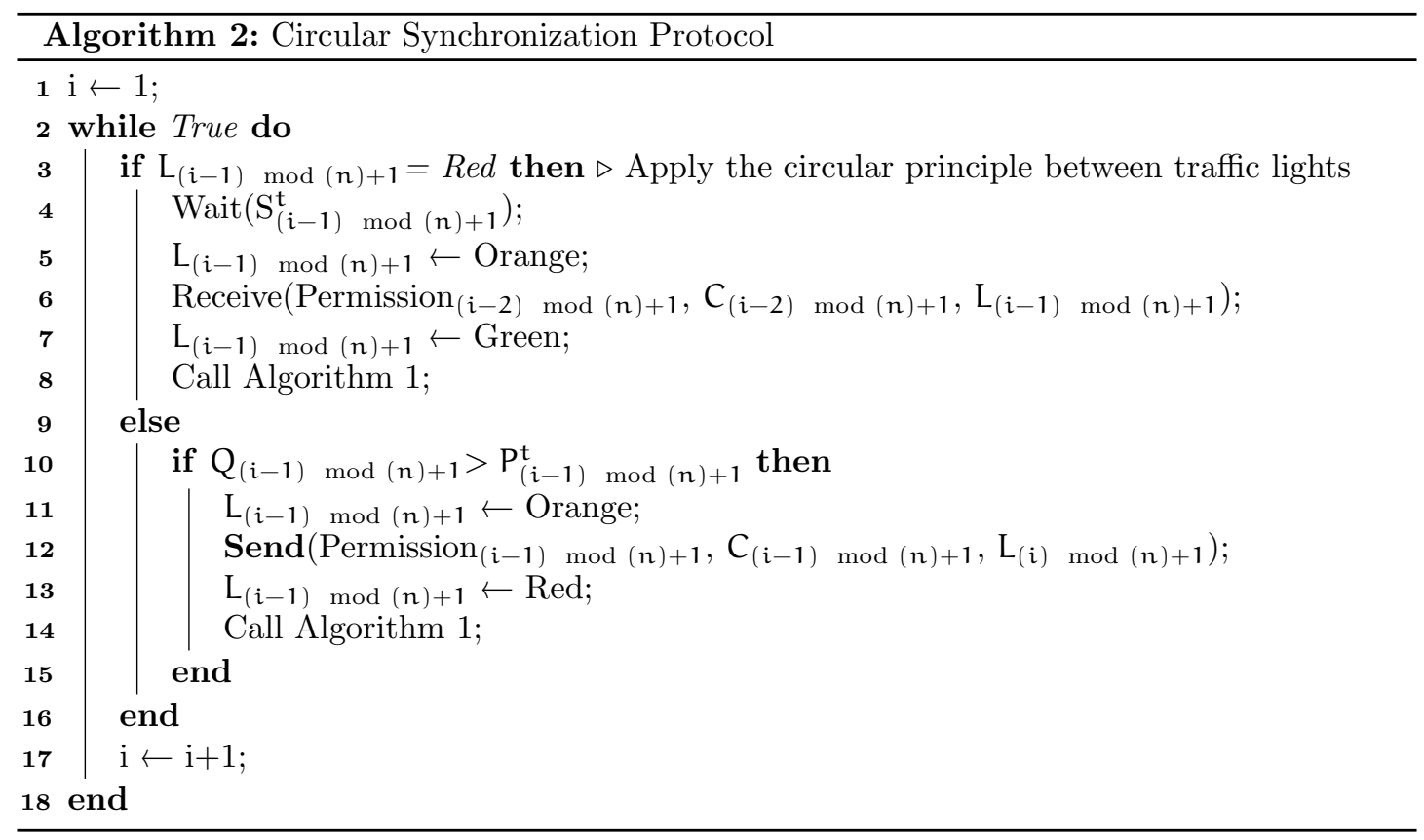

\section{A. Crossing Time Scheduling Using Genetic Algorithm}

Here, we aim to optimize the crossing time of negotiating vehicles assigned to each phase of traffic lights, using the genetic algorithm under the following two objectives: (i) Determinate the effective density of negotiating vehicles supervised by each traffic light $L_{i}$, according to crossing time $C_{i}^{t}$. (ii) Minimize the total waiting time of these vehicles at signalized intersection road. In the genetic algorithm based optimization crossing time scheduling, the chromosome is an integer vector, where each gene represents crossing time duration of each traffic light $L_{i}$ involved in a given intersection. It must satisfy the following condition:

$$
0 \leq \mathrm{C}_{\mathrm{i}}^{\mathrm{t}} \leq \mathrm{T}^{\mathrm{c}}
$$

In this paper, we vary the cycle time $\mathrm{T}^{\mathrm{c}}$ as 80 seconds, 100 seconds, and 120 seconds, which involves that chromosome size is represented as $7 \times n$ bit, where $n$ is the number of traffic lights in signalized intersection. Moreover, the initial population is set to be 50 chromosomes.

In order to determine the effective density of negotiating vehicles, we define the real distance $D$, which represents the maximum coverage of each traffic light $L_{i}$. D allows the traffic lights to monitor a real density $d_{i}^{r}$ of traffic flow in the road intersection. It depends on the cycle time $\mathrm{T}^{\mathrm{c}}$ and given as

$$
\mathrm{D}=\mathrm{S}^{\mathrm{r}} \times \mathrm{T}^{\mathrm{c}}
$$

We take the Equation 2 when $\sum_{j=1}^{d_{i}^{e}} t_{i}\left(v_{j}\right)=\varepsilon$. Hence, the effective distance $D_{i}^{e}\left(v_{l}\right)$ of 
negotiating vehicles supervised by each traffic light $L_{i}$ is calculated as

$$
\mathrm{D}_{i}^{e}\left(v_{l}\right)=\mathrm{C}_{i}^{\mathrm{t}} \times \mathrm{S}^{\mathrm{r}} .
$$

The effective density of negotiating vehicles $d_{i}^{e}$ authorized to cross the signalized intersection is generated dynamically as illustrated in Algorithm 3 . This operation is done according to the total length $l g=\sum_{j=1}^{d_{i}^{e}} l_{j}$ of vehicles, the total safety distance $h=\sum_{j=2}^{d_{i}^{e}} h_{j}$ between vehicles, and $\phi_{i}$ that represents the distance between the first vehicle and the traffic light $L_{i}$.

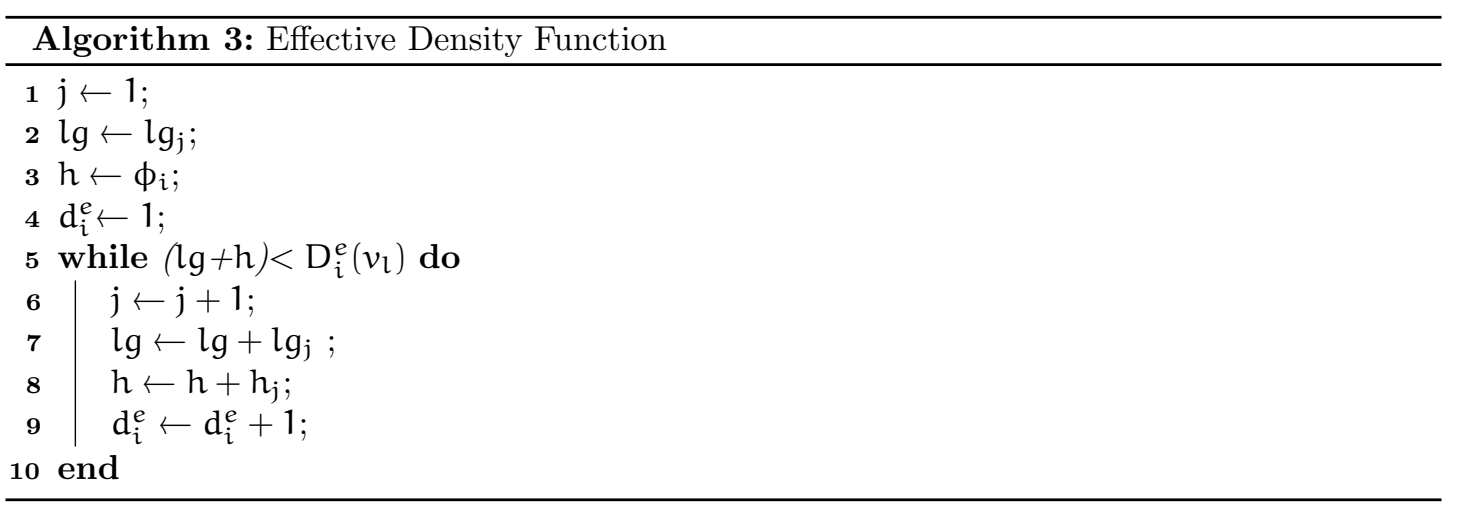

In $G A$, the fitness function tends to minimize the total waiting time $T^{w}$ spent by vehicles at signalized road intersection, such as

$$
\mathrm{T}^{w}=\sum_{i=1}^{n} \sum_{j=1}^{\mathrm{d}_{i}^{\mathrm{e}}} \mathrm{t}_{i}^{w}\left(v_{j}\right),
$$

based on Equation 6 , the waiting time $t_{i}^{w}\left(v_{j}\right)$ spent by a $v_{j}$ at each traffic light $L_{i}$ is measured as

$$
t_{i}^{w}\left(v_{j}\right)=T_{i}^{g}-T_{i}^{a}\left(v_{j}\right)+C_{i}^{t}\left(v_{j}\right),
$$

where $T_{i}^{g}$ is the start time of green light at each $L_{i}, T_{i}^{a}\left(v_{j}\right)$ is the arrival time of $v_{j}$, and $C_{i}^{t}\left(v_{j}\right)$ is the crossing time of each $v_{j}$. The crossing time is calculated as

$$
C_{i}^{t}\left(v_{j}\right)=\frac{D_{i}^{e}\left(v_{j}\right)}{S^{r}}+t_{i}\left(v_{j}\right),
$$

where $D_{i}^{e}\left(v_{j}\right)$ is the effective distance between the $v_{j}$ and the $L_{i}$.

With each change in negotiating vehicle requests, GA regenerates a crossing time and calculates the total waiting time at each iteration. After a set of iterations, if the total waiting time is reduced, then we reiterate. Otherwise, we stop and the fitness limit is set to be the chromosome, that represents the sequence terminates of crossing time giving the minimum of the $T^{w}$.

\section{B. Intelligent Context-Aware Negotiation Protocol}

We propose an intelligent context-aware negotiation protocol (ICANP) illustrated in Algorithm 4. ICANP is able to maximize the number of vehicles that reach their destination. In this protocol, the crossing time $C_{i}^{t}$ assigned at each phase of $L_{i}$ urges the effective density of negotiating vehicles to cross the intersection on one hand, and doesn't block the other conflicting traffic flow in the other hand. Where crossing strategies for negotiating vehicles are differents from those of the circular case, we study in this paper three typical cases as described in Figure 2 . 


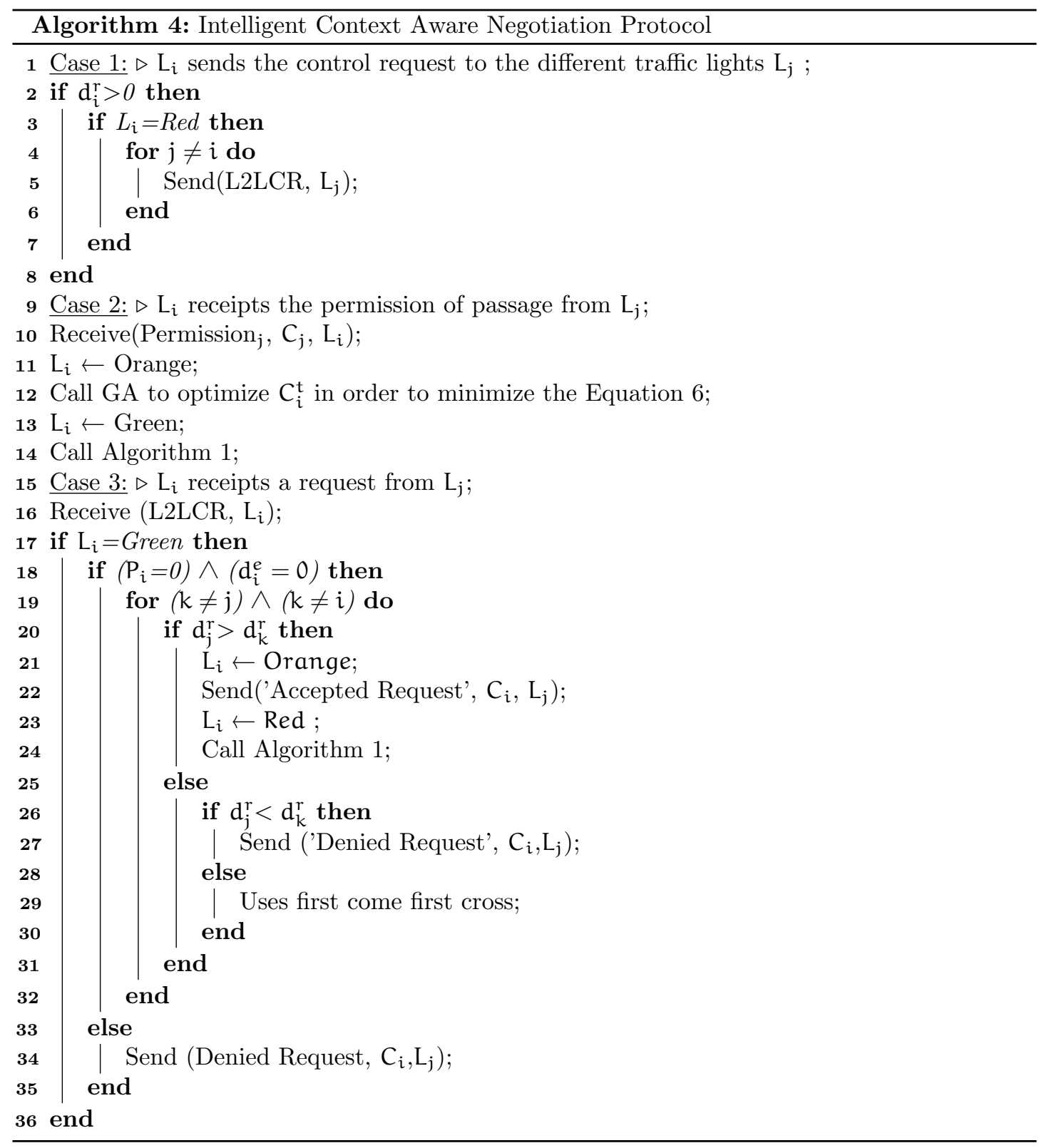

At time $t_{0}$, when the traffic light $L_{1}$ that supervises the group $g_{1}$ of negotiating vehicles is green. Then, it their authorizes to cross the intersection. But, if $\mathrm{L}_{1}$ does not have a green light, it negotiates the traffic light that owns it at this moment. If the solicited traffic light $L_{4}$ has neither priority vehicles $\left(P_{i}=0\right)$, nor of negotiating vehicles $\left(d_{i}^{e}=0\right)$ at this moment, it interrupts the regular vehicles, that are already started to cross the intersection in order to give the green light to the requesting traffic light $\mathrm{L}_{1}$. Besides, each traffic light repeats the negotiation procedure with other traffic lights located in the same intersection for each incoming traffic flow, that contains at least a negotiating vehicle. 


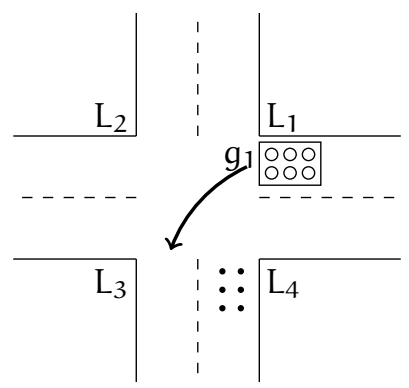

Scenario 0

$t_{0}$

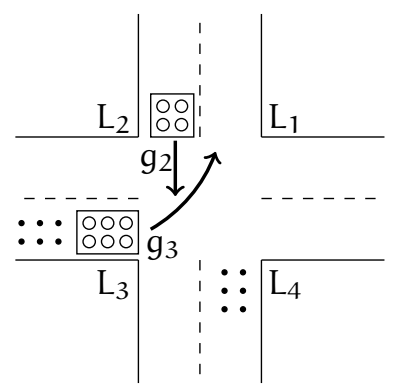

Scenario 1

$t_{1}$

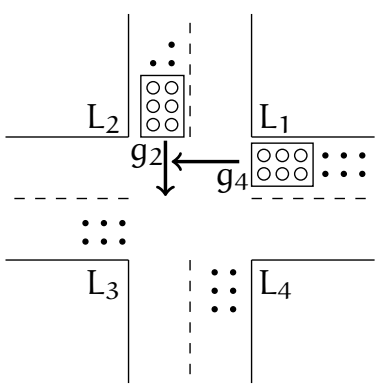

Scenario 2 $t_{2}$

$\circ$ Negotiating vehicles

- Regular vehicles

Figure 2: Conflicting traffic flow scenarios in negotiation case

In scenario 2 , at the moment $t_{1}$, the $g_{2}$ and $g_{3}$ of negotiating vehicles supervised respectively by the $L_{2}$ and $L_{3}$ are detected close at a signalized intersection. In this case, the $g_{3}$ is allowed to cross the intersection first. Indeed, its effective density $\mathrm{d}_{3}^{e}$ is greater than the effective density $\mathrm{d}_{2}^{e}$ of $\mathrm{g}_{2}$, after having carried out a negotiation procedure if necessary. Finally, in scenario 3 at the moment $t_{3}$, two new negotiating vehicles joined the group $g_{2}$. Moreover, another group $g_{4}$ arrived at the signalized intersection, according to the proposed negotiation protocol we apply first come, first cross, in which a greater preference is assigned to $\mathrm{g}_{2}$.

\section{Negotiation Protocol Based on Reputation}

The vehicles communicate directly with the traffic light by exchanging the control requests V2LNR. Each vehicle is controlled by its driver, in which it personalizes at the starting the position of its destination and travel urgency. A dishonest driver could cross the intersection by sending various false information to the traffic light. Therefore, it can cause accidents and increase the waiting time of honest drivers at the signalized intersection. Here, we evaluate the behavior of all negotiating vehicles at signalised intersection. For this reason, we introduce a reputation model to evaluate the reliability of the negotiation requests V2LNR exchanged between the traffic light and drivers. This model takes into account the context of driver, which consists of two parts: (i) The static context that is represented by his personal information, such as $\operatorname{VID}_{i}^{j}$, DID ${ }^{j}$, etc. (ii) The dynamic context that consists of $\mathrm{RD}^{j}, \mathrm{DS}^{j}$ and $\mathrm{U}^{j}$. TheNBPR aims to reduce to a minimum the effect of incoming dishonest drivers at signalized intersection. Figure 3 shows the proposed reputation model in traffic light (RMTL). RMTL uses the reputation values to evaluate the drivers behaviors before allowing them to cross at the signalized intersection. The negotiating vehicles are represented by context-based reputation. However, priority vehicles are represented by role-based reputation.

In our work, we suppose a reputation interval between 0 and 1 , where 0 is the totally dishonest and 1 is fully trustworthy. Furthermore, each vehicle has a reputation value belonging to this interval, which is stored in the traffic light. Our reputation model is based on the following phases:

1. Passage verification: At the arrival of negotiating vehicles, RMTL searches the reputation of drivers through direct and indirect strategy as illustrated in Equation 9 : (1) In direct strategy, the traffic light $L_{i}$ calculates the vehicle reputation value based on its local experience, which represents the history of previous interactions with the same vehicle. If the 
vehicle behaves correctly in the past, so, it has a reputation value, that allows it to cross at the intersection. (2) In indirect strategy, the vehicles can communicate in VANET via $\mathrm{V} 2 \mathrm{~V}$ for different reasons (guidance help, warning messages, local danger, change in message integrity, etc.). On this view, the traffic light $\mathrm{L}_{i}$ searches to have the reputation values of vehicles either through their behavior in VANET via TCM or recovered from the neighboring traffic lights through the reporting messages, such as

$$
R_{i}^{j}=\alpha \cdot r_{i}^{(1, j)}+(1-\alpha) \cdot\left(\left(\beta \cdot r_{i}^{(2, j)}+(1-\beta) \cdot r_{i}^{(3, j)}\right)\right),
$$

where $R_{i}^{j}$ represents the reputation value of each $v_{j}$ supervised by the $L_{i}, \alpha$ and $\beta$ are a weighted factor to maintain the value of reputation $(\alpha, \beta \in\{0,1\}), r_{i}^{(1, j)}$ is the local reputation of $v_{j}$ in traffic light $L_{i}, r_{i}^{(2, j)}$ represents the reputation of $v_{j}$ in VANET, and $r_{i}^{(3, j)}$ represents the reputation of $v_{j}$ in neighboring traffic lights.

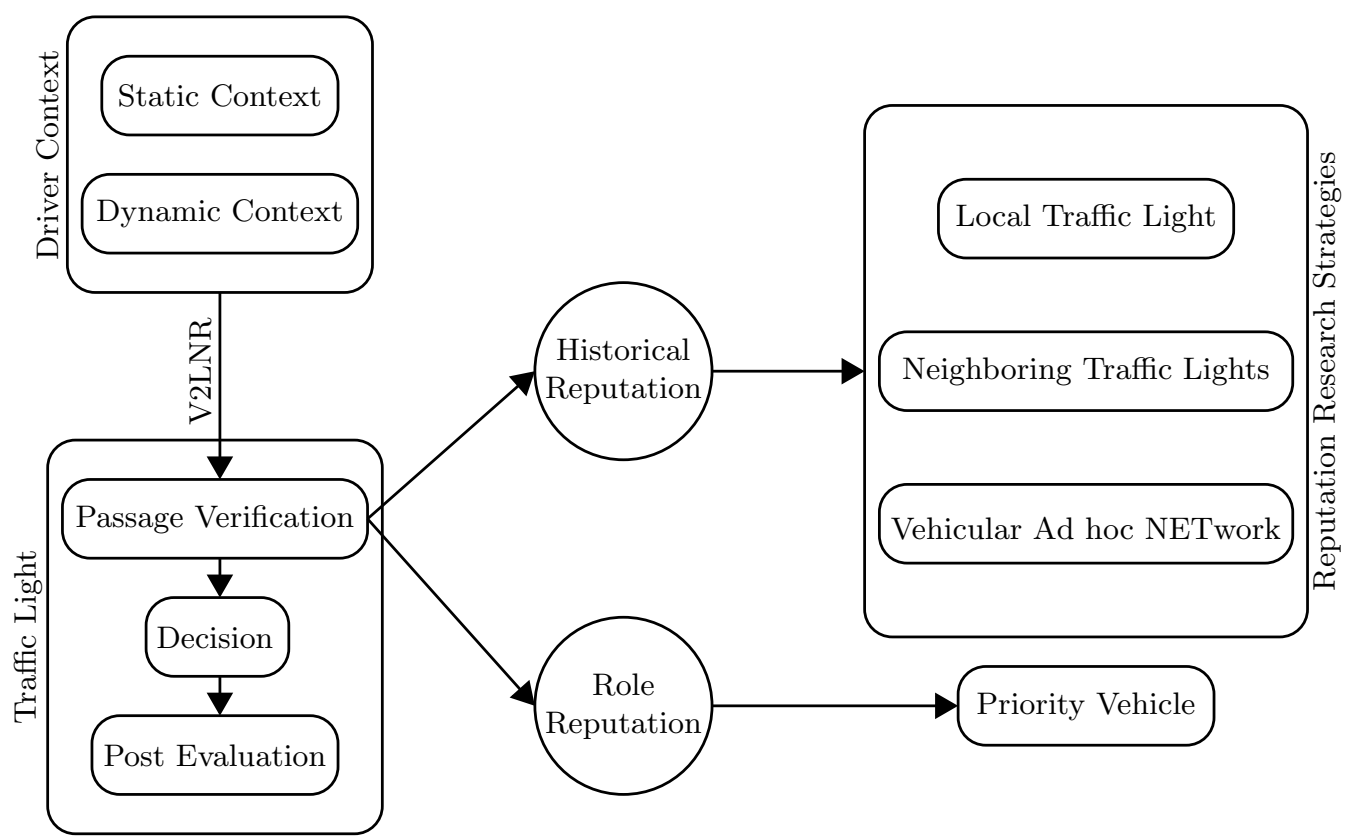

Figure 3: Reputation model in traffic light

According to the Equation 9 we classify the vehicles into three categories shown in Figure 4 Dishonest vehicles, honest vehicles and reputed vehicles. Dishonest vehicles have a reputation value below than a given threshold $\gamma\left(0 \leq R_{i}^{j}<\gamma\right)$. Honest vehicles have a reputation value between $\gamma$ and $\delta\left(\gamma \leq R_{i}^{j}<\delta\right)$. They can negotiate their crossing time. Reputed vehicles have a high reputation value between $\delta$ and $1\left(\delta \leq R_{i}^{j} \leq 1\right)$. They can pass directly without negotiation.

Given the random behavior and position of dishonest vehicles in conflicting traffic flow, the negotiation protocol based on reputation(NPBR) as shown in Algorithm6, uses the reputation value $R_{i}^{j}$ of each negotiating vehicle. NBPR calculates the volume of dishonest vehicles $V_{i}^{D}$ as being illustrated in the Algorithm 5 . 


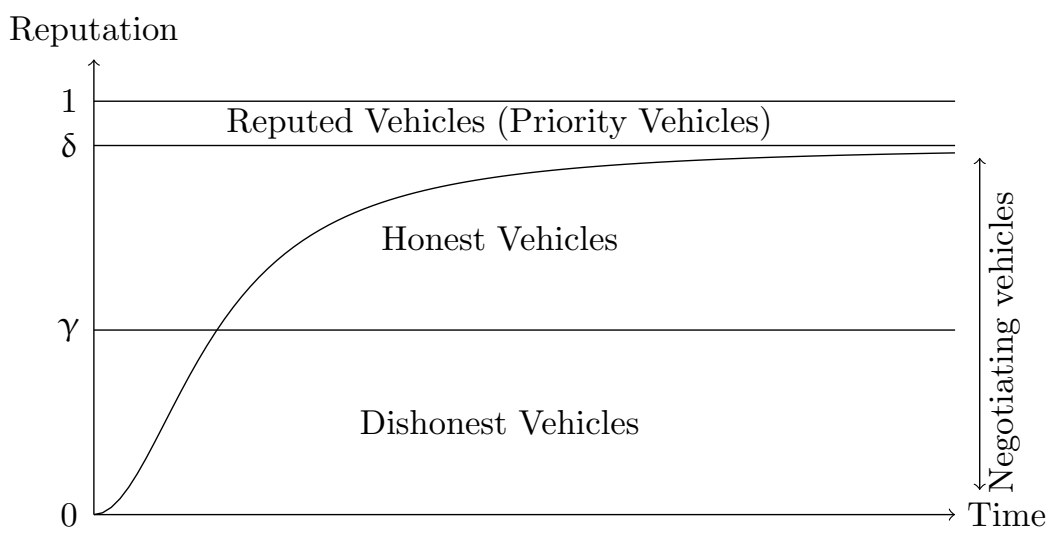

Figure 4: Reputation levels for different types of vehicles

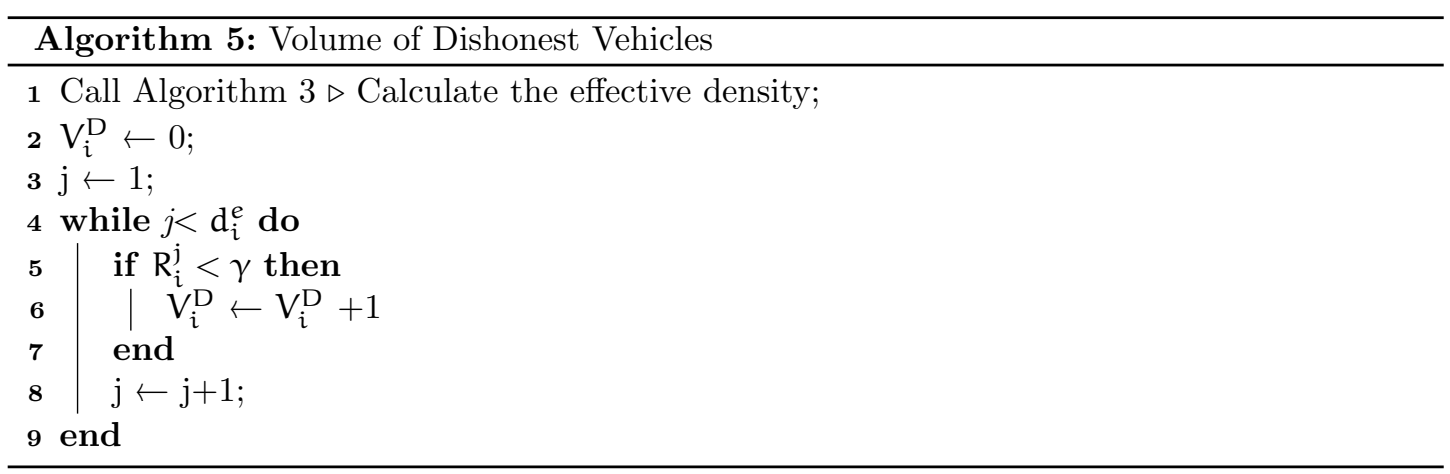

NPBR in case 2 , uses GA to optimize the crossing time of effective density $d_{i}^{e}$, that has a minimum volume of dishonest vehicles $\mathrm{V}_{i}^{\mathrm{D}}$. Therefore, the total waiting time $T^{w}$ of the negotiating vehicles is minimized such as

$$
T^{w}=\sum_{i=1}^{n}\left(\sum_{j=1}^{d_{i}^{e}-V_{i}^{D}} t_{i}^{w}\left(v_{j}^{H}\right)+\sum_{k=1}^{V_{i}^{D}} C_{i}^{t}\left(v_{k}^{D}\right)\right),
$$

where $t_{i}^{w}\left(v_{j}^{\mathrm{H}}\right)$ represents the waiting time spent by each honest $v_{j}$, achieved in each $\mathrm{L}_{i}$, and $C_{i}^{t}\left(v_{k}^{D}\right)$ is the crossing time of each dishonest vehicle $k$ achieved in each traffic light $L_{i}$.

2. Decision: NBPR scheduling algorithm in case 3 , prioritizes the real density $d_{i}^{r}$ of conflicting traffic flow, that has a heaviest density of honest vehicles $d_{i}^{(r, H)}$ supervised by each $L_{i}$ to reach their destination as soon as possible.

3. Post evaluation: The traffic light $\mathrm{L}_{i}$ compares the requested direction $R \mathrm{D}^{j}$ of each negotiating $v_{j}$, with crossing direction $\mathrm{CD}^{j}$ selected after the passage at signalized intersection. If they are identical, then the reputation value $R_{i}^{j}$ of $v_{j}$ increases by $\theta$, otherwise it decreases by the same value. The traffic light stores the $v_{j}$ as honest, when its reputation value is greater than or equal to threshold $\gamma$. If the reputation of negotiating $v_{j}$ is less than the threshold $\gamma$. Therefore, the traffic light $L_{i}$ stores it as dishonest. In addition, if at a certain time, the $R_{i}^{j}$ of $v_{j}$ continues to decrease until below 0 . Then, the $v_{j}$ is filtered in a blacklist. The principle 
of post evaluation is detailed in the Algorithm 7

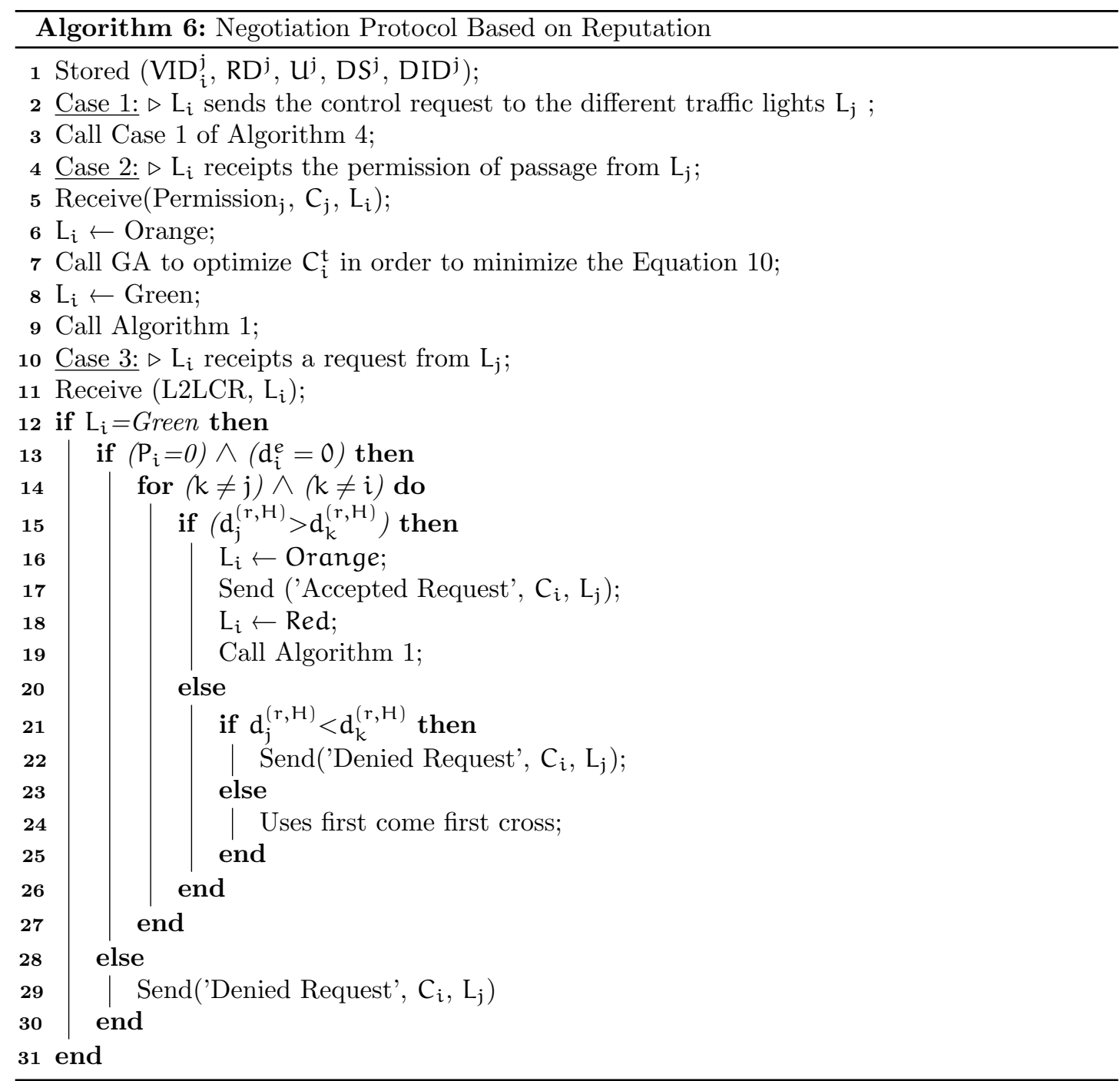

\subsubsection{Priority Case}

As illustrated in Algorithm 7, the priority vehicles are the set of reputed vehicles without negotiation, having a reputation value in $[\delta, 1]$. They follow the same principle of negotiating vehicles, that is illustrated in scenario 0 and 1 of Figure 2 But also, we propose three other scenarios, considering the presence of negotiating vehicles as illustrated in Figure 5 , and described in Algorithm 8 .

In scenario 0 , at the moment $t_{0}$, the group $g_{1}$ and $g_{2}$ of priority vehicles are close to signalized road intersection. They have the same density $\left(d_{1}^{(r, p)}=d_{2}^{(r, p)}\right) . g_{1}$ proceed first, if its average reputation $\left(R_{1}^{A}\right)$ is greater than that of group $g_{2}\left(R_{2}^{A}\right)$. Here, $R_{i}^{A}$ is calculated using $d_{i}^{(r, p)}$, such as

$$
R_{i}^{A}=\frac{\sum_{j=1}^{d_{i}^{(r, p)}} R_{i}^{j}}{d_{i}^{(r, p)}},
$$


at the time $t_{1}$, both groups $g_{3}$ and $g_{4}$ have the same density $\left(d_{3}^{(r, p)}=d_{4}^{(r, p)}\right)$, and the same average reputation $\left(R_{3}^{A}=R_{4}^{A}\right)$. Then, $g_{3}$ crosses the intersection first because the density of negotiating vehicles group, that follows it, is greater than that of $\mathbf{g}_{4}$. Finally, in scenario 2 , the groups $g_{5}$ and $g_{6}$ have also the same density and the same average reputation. $g_{5}$ schedules the signalized road intersection first, because its negotiating vehicles group which follows it, has a minimum of $\mathrm{V}_{1}^{\mathrm{D}}$ than the group $\mathrm{g}_{6}$.

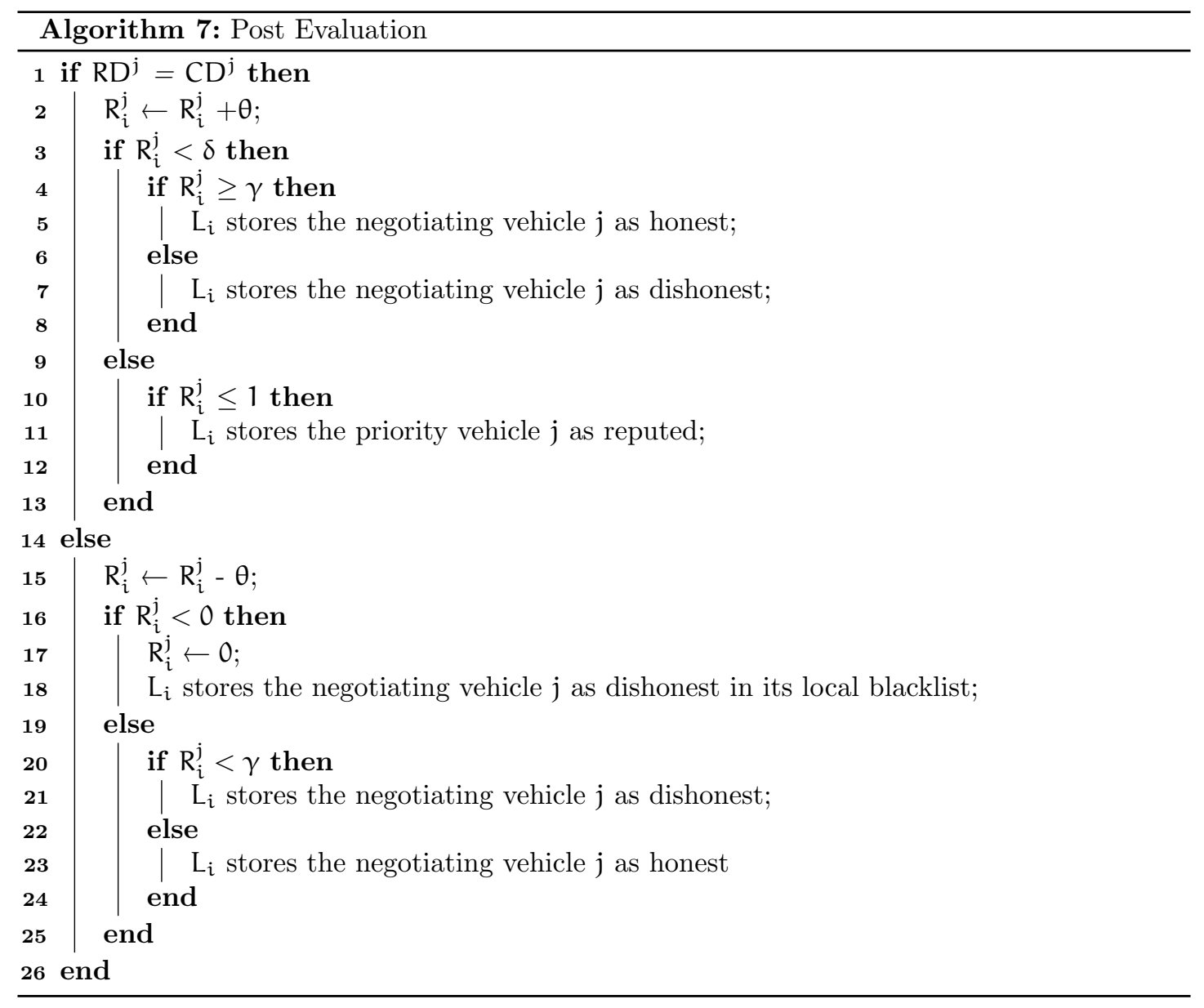




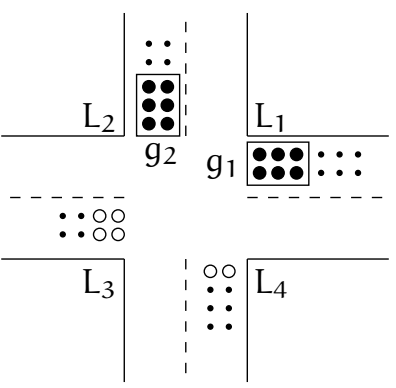

Scenario 0

$t_{0}$

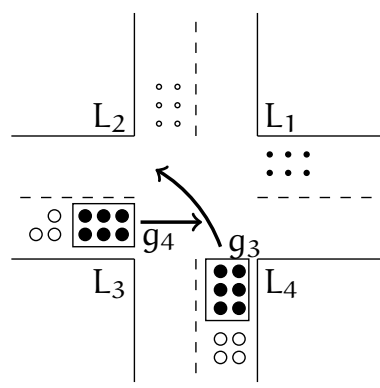

Scenario 1

$t_{1}$

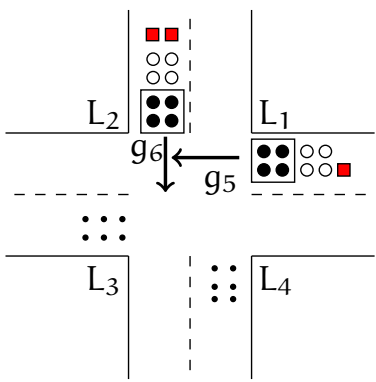

Scenario 2

$t_{2}$

- Priority vehicles

- Negotiating vehicles

- Regular vehicles

- Dishonest vehicles

Figure 5: Conflicting traffic flow scenarios in priority case

\section{Simulation Results}

In this section, first, we study the intersection used in our experiments. After this, we present details about the used hardware and software configuration for simulation purposes. Then, we simulate the proposed protocols, evaluate their performance and compare it with related works [1, 2] to demonstrate their effectivity. Later, we present a analytical report on the computational complexity required for our protocols.

\subsection{Studied Intersection}

The studied system is a road network consisting of a major signalized intersection located in urban areas of approximately 1,2 km of Iris city in Bejaia (Algeria). Figure 6 shows the selected area of the city obtained from Google Map view exported form OpenStreetMap (OSM). This intersection has been chosen since it exhibit over-saturation and extreme traffic conditions. It is composed of 4-leg road that has four traffic lights.

We consider that the day is classified in peak hours, when the demand of negotiating vehicles reaches its maximum compared to other non-peak hours. The experiments discuss the traffic scenarios related to real situations for each interval during 12hours. Simulation time was fixed at $2500 \mathrm{~s}$ for intersection under study. This time was determined as a maximum average waiting time spent by vehicle, from its arrival date at signalized intersection circulating with a recommended speed of $40 \mathrm{~km} / \mathrm{h}$. Table 3 shows the number of negotiating vehicles in circulation at intersection under study of one-day, which are collected according to our practical experience in the area. This section discusses the simulation experiments. Here, we focused during peak intervals as $[7: 30-9: 30],[11: 30-13: 30]$ and $[15: 30-19: 30]$ compared with non peak intervals as $[9: 30-11: 30],[13: 30-15: 30]$ and $[17: 30-19: 30]$. 


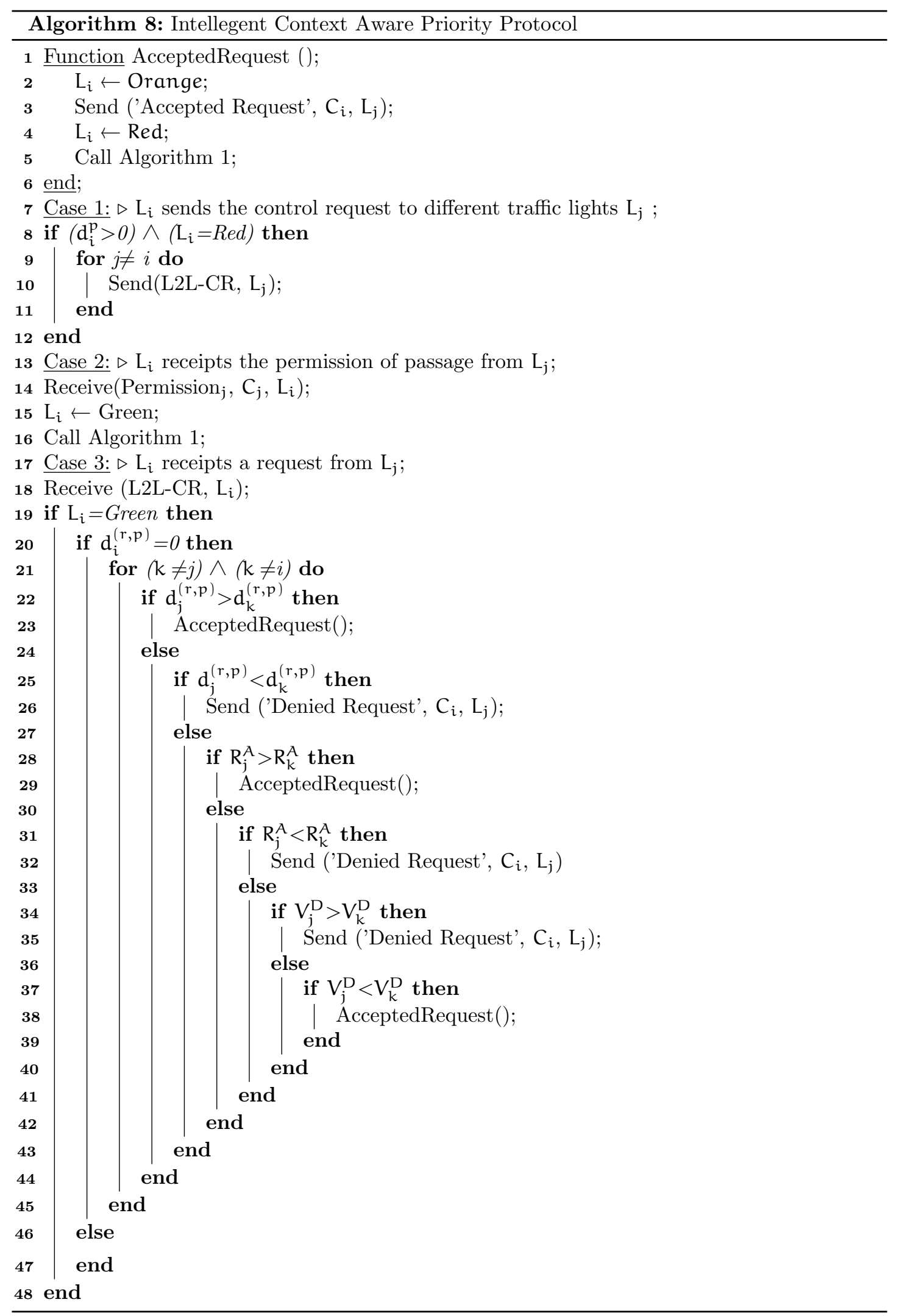




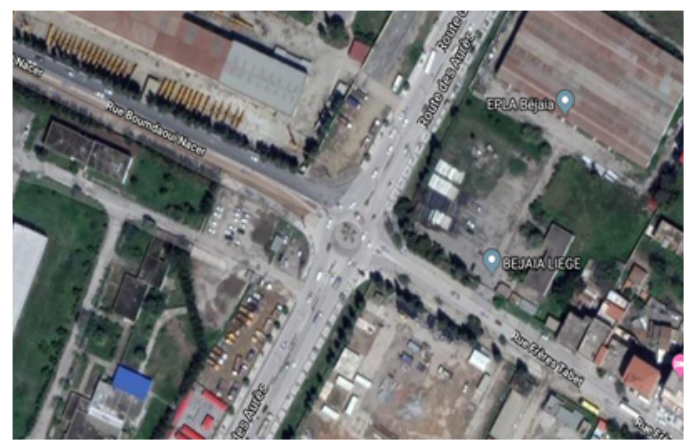

(a)

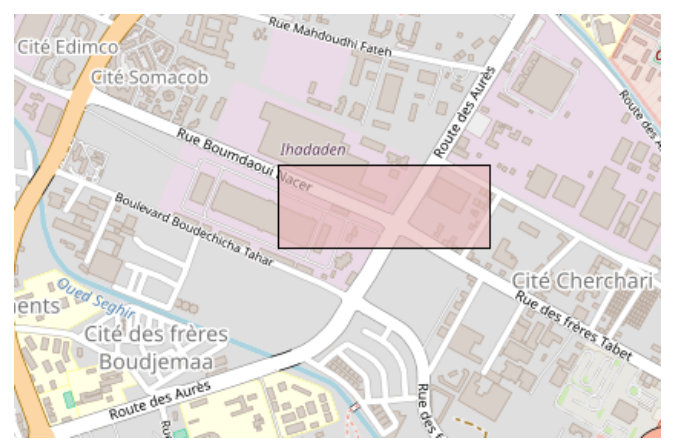

(b)

Figure 6: Intersection used in simulation. (a) Google Map View. (b) OpenStreetMaps View

\begin{tabular}{lc}
\hline Time interval & Number of vehicles \\
\hline $7: 30-9: 30$ & 600 \\
$9: 30-11: 30$ & 150 \\
$11: 30-13: 30$ & 500 \\
$13: 30-15: 30$ & 200 \\
$15: 30-17: 30$ & 700 \\
$17: 30-19: 30$ & 100 \\
\hline
\end{tabular}

Table 3: Vehicles density in circulation at intersection under study

\subsection{Simulation Setting}

We have implemented our protocols with Java jdk - 8u45 - windows - x64 using eclipse JUno version 4.9.0. Furthermore, the experiments were performed on a laptop. The laptop is equipped with Intel Core2(TM)i5 - $4210 \mathrm{U}$ at $2.40 \mathrm{GHz}$ and $6 \mathrm{~GB}$ memory running windows 10 operating system. For each interval, we have carried out 6 independent runs of GA. The population size was set to 50 chromosomes. We performed 1200 optimization iterations, which are: 300 iterations in $[7: 30-9: 30], 100$ iterations in $[11: 30-13: 30], 250$ iterations in [15:30-19:30], 150 iterations in [9:30-11:30], 350 iterations in $[13: 30-15: 30]$, and 50 iterations in $[17: 30-19: 30]$. The latter are the resulting in a total of 60000 solutions in all the intervals. The probabilities of crossing and mutation operators are fixed for all generations and were set to $P_{c}=0.6, P_{m}=0.4$. Additionally, in order to prove the efficiency of the proposed protocols, we have implemented three algorithms also in Java to establish comparisons, including a circular shown in Algorithm 2, ITLC [1] and CATLS 2, respectively.

\subsection{Performance Evaluation}

In order to evaluate the performances of the proposed protocols, we express the congestion by the total waiting time spent by vehicles in the system. A total waiting time of vehicles trying to cross the signalized intersection at the different time intervals of day is calculated for each synchronization protocol (ICANP, NPBR, and ICAPP). A comparison series have been performed for different protocols cited in the literature. A first comparison concerns ITLC, which claimed having best performance over all the previously proposed traffic light scheduling algorithms. Second, we compare ICAPP to CATLS by considering the scenarios described in Figure 5. CATLS is chosen because it is a traffic light scheduling algorithm, which considers 


\begin{tabular}{ll}
\hline Parameter & Value \\
\hline Type of intersection & 4 -leg road intersection \\
Simulation time & 2100 seconds \\
Total number of negotiating vehicles & 2250 \\
Number of traffic light $(\mathrm{n})$ & 4 \\
$\mathrm{Q}_{\mathrm{i}}$ in circular case & 30 seconds \\
$\mathrm{T}^{\mathrm{c}}$ & 120 seconds \\
Crossing time & $\mathrm{C}_{\mathrm{i}}^{\mathrm{t}}$ \\
Recommended speed & $40 \mathrm{~km} / \mathrm{h}$ \\
Effective density & $\mathrm{d}_{\mathrm{i}}^{e}$ \\
Population size & 50 \\
Maximum number of evaluation & 60000 \\
$\mathrm{P}_{\mathrm{c}}$ & 0.6 \\
$\mathrm{P}_{\mathrm{m}}$ & 0.4 \\
$\mathrm{D}$ & 500 \\
$\delta$ & 0.9 \\
$\gamma$ & 0.5 \\
Priority vehicles rate & $5 \%, 15 \%, 25 \%, 30 \%$ \\
Negotiating vehicles rate & $30 \%$ \\
Dishonest vehicles rate & $0 \%, 40 \%, 80 \%$ \\
$\cdot$ & $\mathrm{Multiplication}$ operation \\
$\mathrm{N}$ & $\mathrm{d}_{\mathrm{i}}^{e}$ \\
\hline
\end{tabular}

Table 4: Simulation parameters

the emergency vehicle. Moreover, we have analyzed the direct impact of the proposed protocols over the environmental factors as $\mathrm{CO}_{2}$. The parameters used during the simulation are given in the Table 4 .

\section{A) Performances of ICANP}

At the beginning of our study, we are interested in the total waiting time of negotiating vehicles compared with circular synchronization protocol and ITLC. As illustrated in Figure 7. the ICANP can reduce by $65 \%$, on average, the total waiting time of negotiating vehicles at intersection compared with the circular synchronization protocol and by $42 \%$ compared with ITLC, especially in the peak interval $[7: 30-9: 30],[11: 30-13: 30]$ and $[15: 30-19: 30]$. This is due to the fact that the ICANP uses double optimization. First, it assigns the green light to traffic light, which supervises a maximum real density of negotiating vehicles, thereby reducing congestion. Second, the GA is run for 1200 iteration . It takes a crossing time to dynamically calculate the effective density of negotiating vehicles trying to cross the intersection. The GA minimizes their total waiting time. In this experiment, we consider that all negotiating vehicles are honest with the volume of dishonest vehicles $\mathrm{V}^{\mathrm{D}}=0 \%$, this is our reference case.

\section{B) Effect of incoming dishonest vehicles on congestion}

Dishonest vehicles can send wrong negotiation requests at traffic light, that supervised them to cross the intersection fast. The purpose of this experiment is to quantify the effect of dishonest vehicles, that may have on the waiting time of negotiating vehicles at signalized intersection. So, we vary among the negotiating vehicles, their volume $\mathrm{V}^{\mathrm{D}}$ is distributed at signalized intersection, the result is shown in Figure 8. We note that, the total waiting time of the negotiating vehicles increases according to the volume of dishonest vehicles. In peak 
interval, the total waiting time increasing by $30 \%$ with $\mathrm{V}^{\mathrm{D}}=80 \%$ compared with $\mathrm{V}^{\mathrm{D}}=40 \%$, which proves that the dishonest vehicles can cause the congestion, thus motivating the use of NPBR, in this work to prevent their impacts.

\section{C) Performances of NPBR}

We measure the performances of NPBR by observing how well it can handle misleading negotiation requests sent by dishonest vehicles. In this simulation, we set the volume of dishonest vehicles $\mathrm{V}^{\mathrm{D}}=80 \%$ at the intersection. As we can see from the Figure 9, NPBR decreases the total waiting time of honest negotiating vehicles by $45 \%$ in peak hours compared to ICANP, and by $64 \%$ compared to ITLC. This is justified by the fact that NPBR prioritizes the traffic flow, which has a greater density of honest negotiating vehicles in real distance of each traffic light. In addition, it uses GA to calculate the effective density of negotiating vehicles having a minimum value of $\mathrm{V}^{\mathrm{D}}$. Here, the volume of dishonest vehicles is calculated by each traffic light using the historical reputation. However, in ITLC, the traffic flow that obtains the highest density is scheduled to pass the road intersection first, without considering the reputation of vehicle.

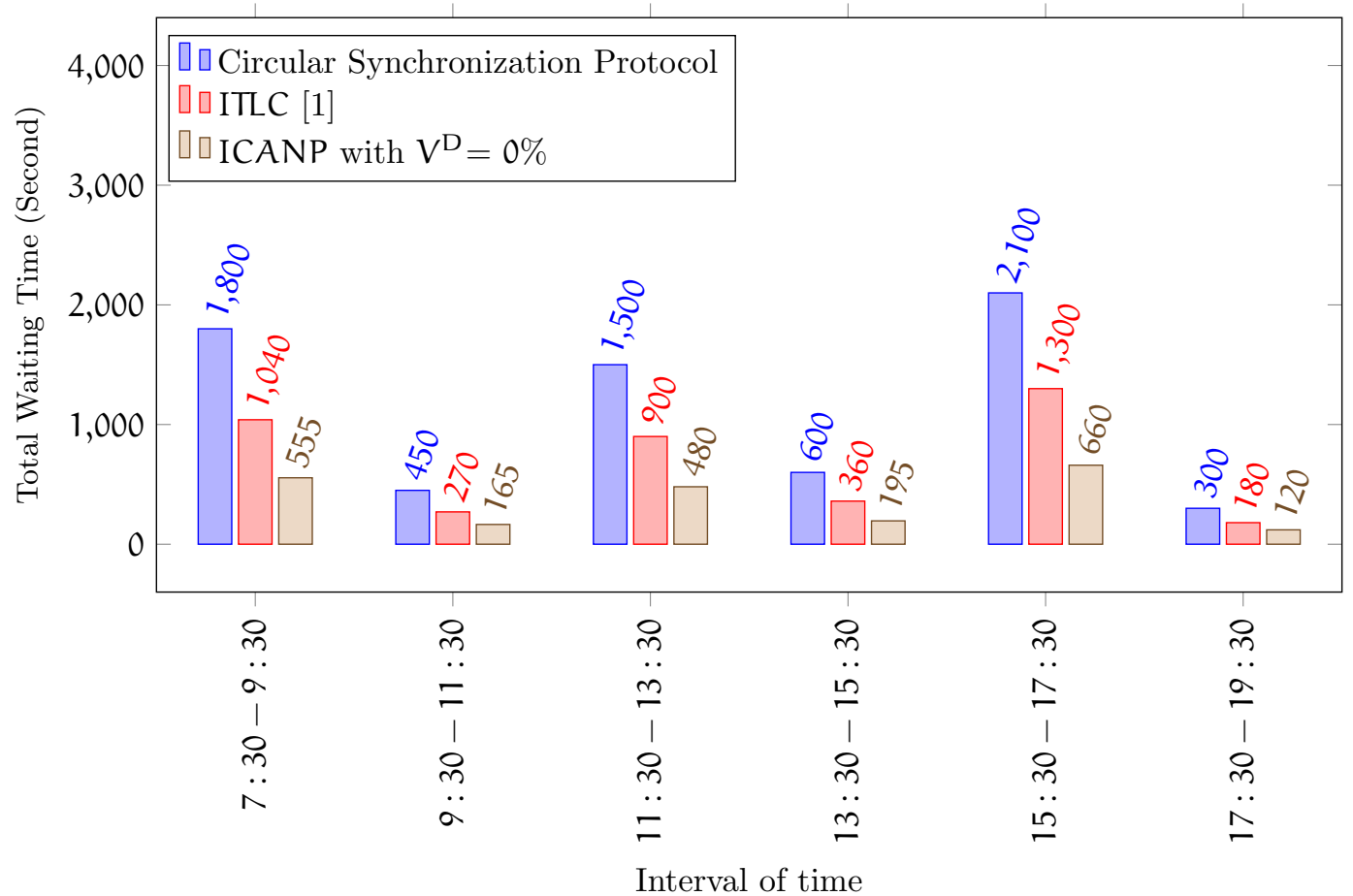

Figure 7: Total waiting time of negotiating vehicles in ICANP compared with circular and ITLC [1] 


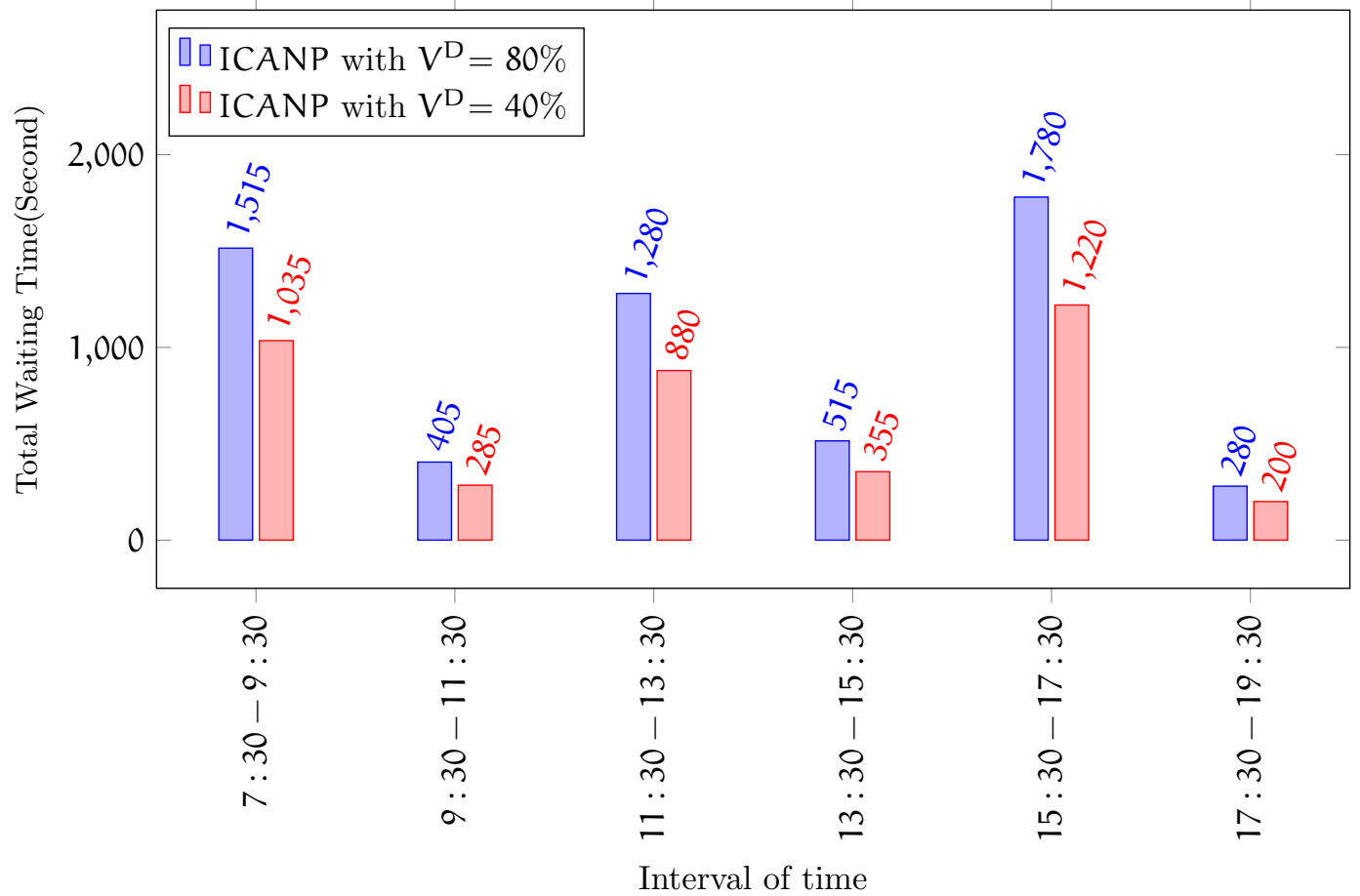

Figure 8: Effect of dishonest vehicles on the total waiting time of negotiating vehicles

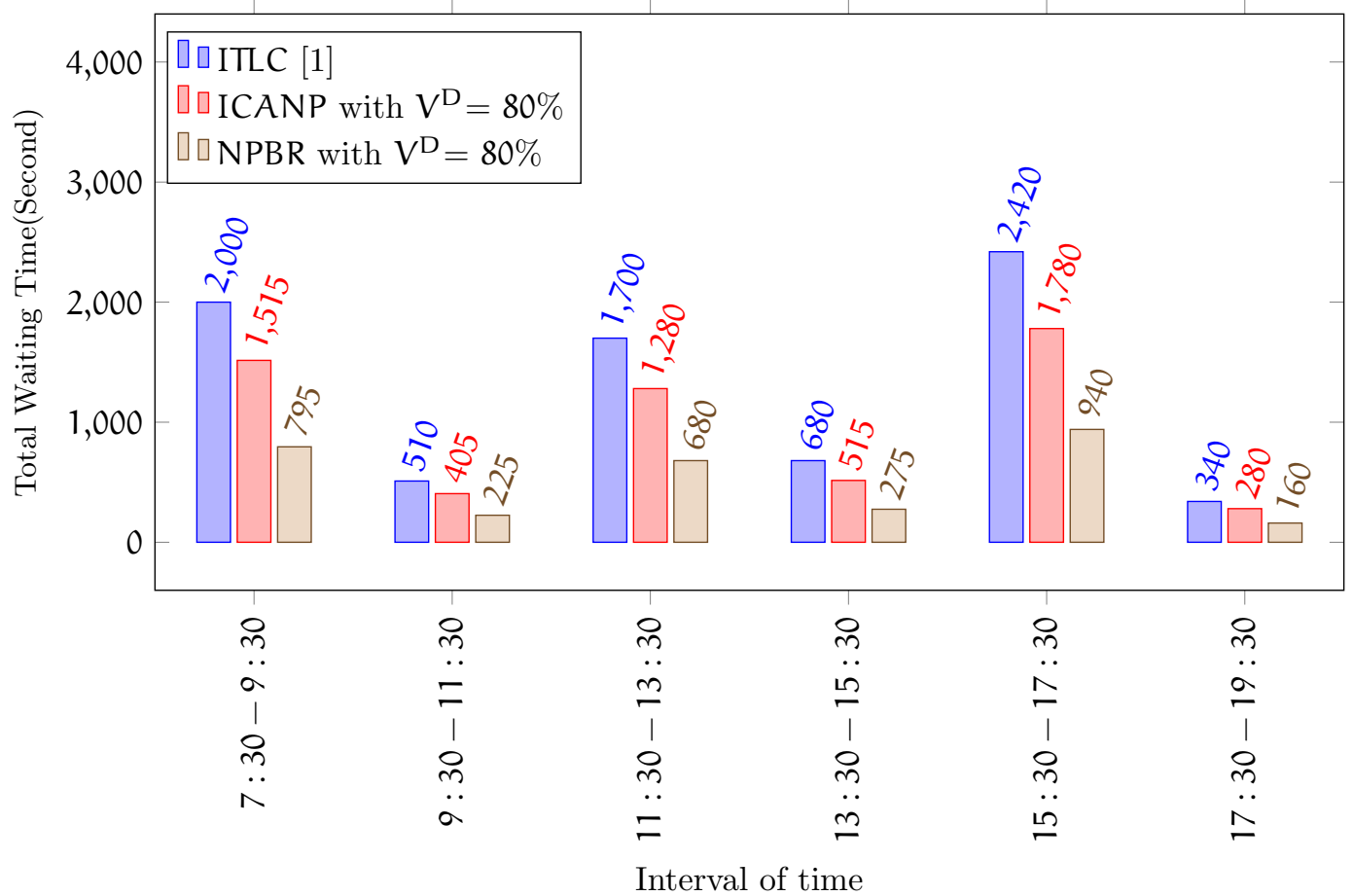

Figure 9: Total waiting time of honest negotiating vehicles in ICANP, NPBR and ITLC [1] 


\section{D) Performances of ICAPP}

Here, we have considered the scenario where many priority vehicles reach the same signalized road intersection simultaneously. Then, we have compared the performances of our protocol ICAPP with CATLS 2 in Figure 10. The results demonstrate that our designed protocol reduce by $32 \%$ the total waiting time of priority vehicles than CATLS. Indeed, ICAPP allow all priority vehicles supervised by each $\mathrm{L}_{i}$ to cross the intersection without interruption. However, CATLS interrupts the green time of lowest priority vehicles, in order to allow highest priority vehicles to past fast.

\section{E) Effect of priority vehicles on ICANP}

In this experiment, we have simulated the scenario 1 of Figure 5 in order to prove the effect of priority vehicles on the total waiting time of negotiating vehicles. For this reason, we combine the negotiating vehicles with different percentage of priority vehicles at the intersection, that are supposed to be always trustworthy. The value of their role-based reputation is between $\delta=0.9$ and 1 . Besides, we have measured the total waiting time of negotiating vehicles, that follow the group $g_{4}$ and $g_{3}$ of priority vehicles. The obtained results are presented in Figure 11. As we can see, ICANP with $30 \%$ of priority vehicles increase by $20 \%$ the total waiting time of negotiating vehicles compared to ICANP with $5 \%$ of priority vehicles.

F) Effect of negotiating vehicles on ICAPP

As the previous experiment, here, we have calculated the total waiting time of priority vehicles $g_{4}$ illustrated in scenario 1 of Figure 5, the results are presented in Figure 12 As we can see, ICAPP with $30 \%$ of negotiating vehicles slightly increase by $25 \%$ the total waiting time of priority vehicles. The reason is that the priority is given a priority vehicles, which have de heaviest density of negotiating vehicles that follow them.

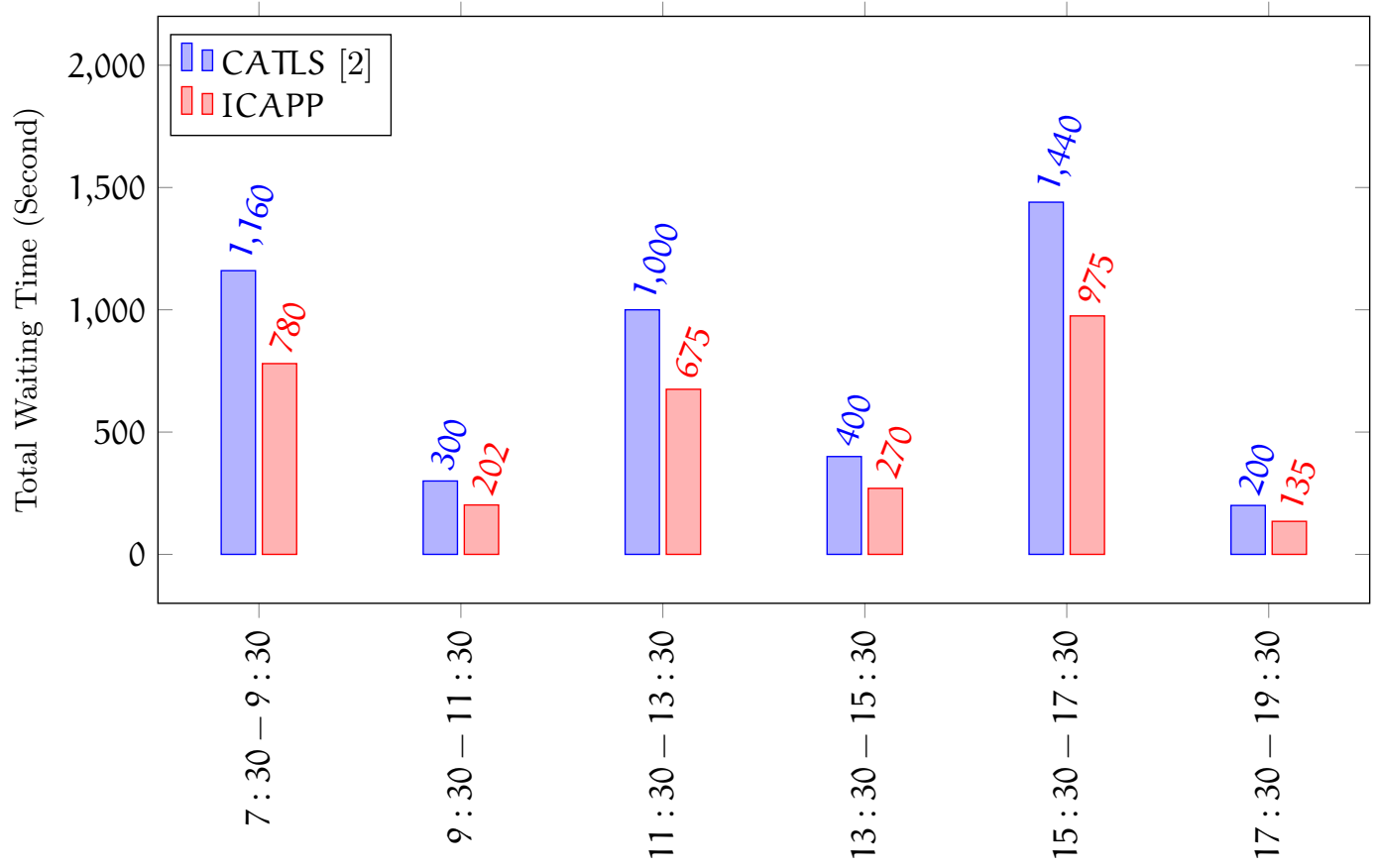

Interval of time

Figure 10: Total waiting time of priority vehicles in ICAPP compared with CATLS 2] 


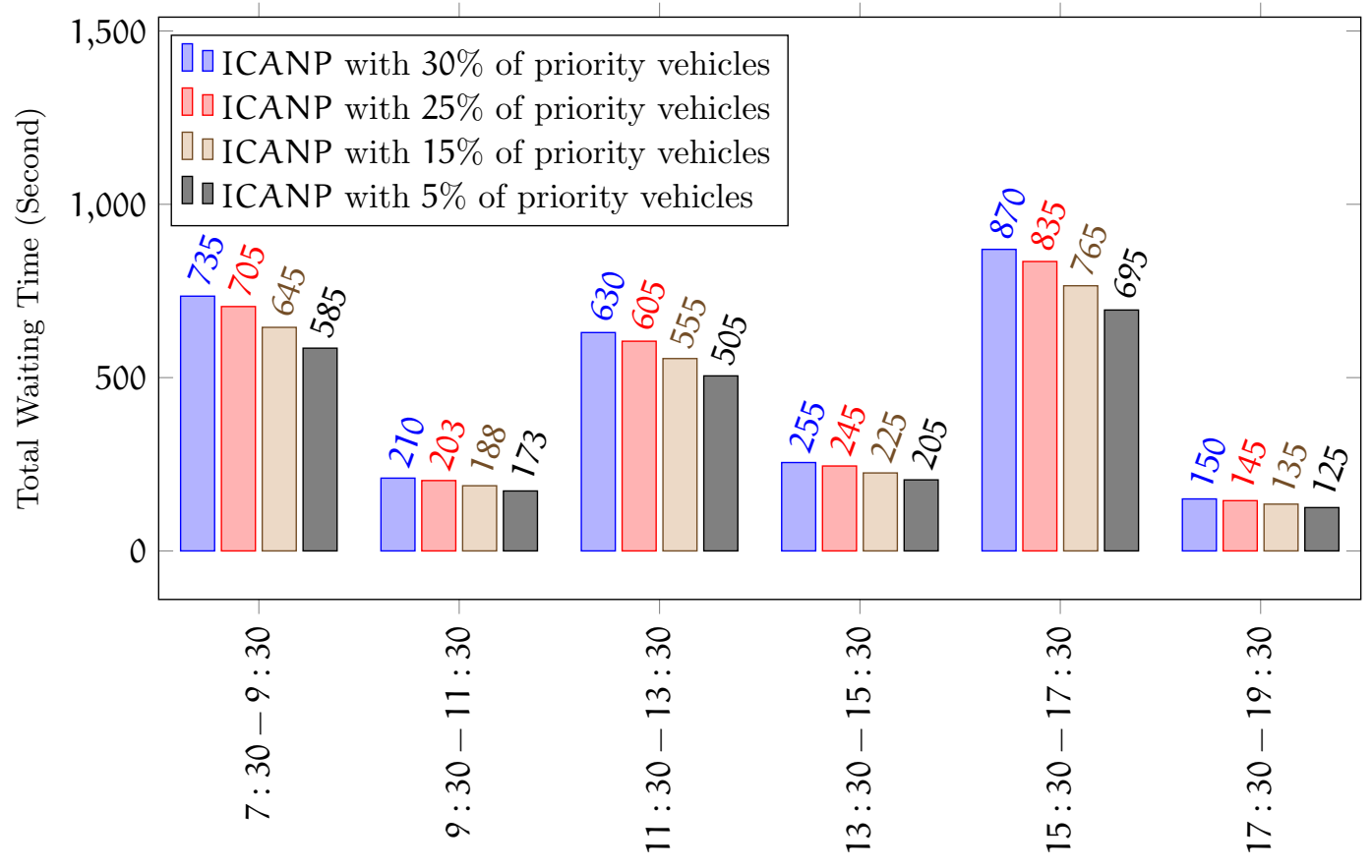

Interval of time

Figure 11: Total waiting time of negotiating vehicles with priority vehicles

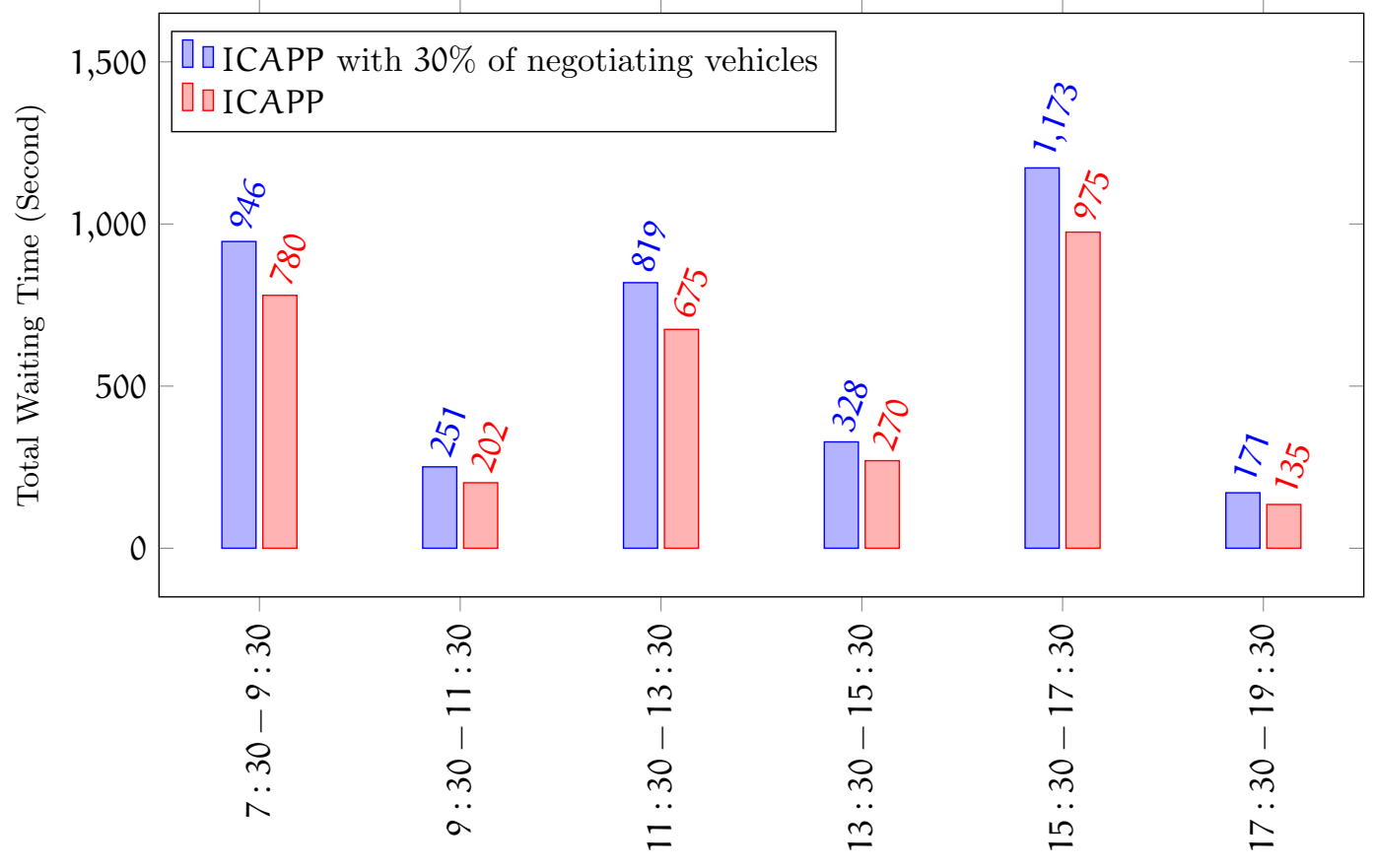

Interval of time

Figure 12: Total waiting time of priority vehicles with negotiating vehicles 


\section{G) Effect of dishonest vehicles on ICAPP}

We test the impact of dishonest vehicles on the total waiting time of priority vehicles as illustrated in scenario 2 of Figure 5. In this context, we introduce $30 \%$ of negotiating vehicles, and we set the volume of dishonest vehicles, i.e., 40\%. As shown in the Figure 13. ICAPP decreases by $30 \%$ the total waiting time of priority vehicles compared to ICAPP with $30 \%$ of negotiating vehicles and $v_{\mathrm{D}}=40 \%$. Consequently, the dishonest vehicles affect slightly the total waiting time of priority vehicles.

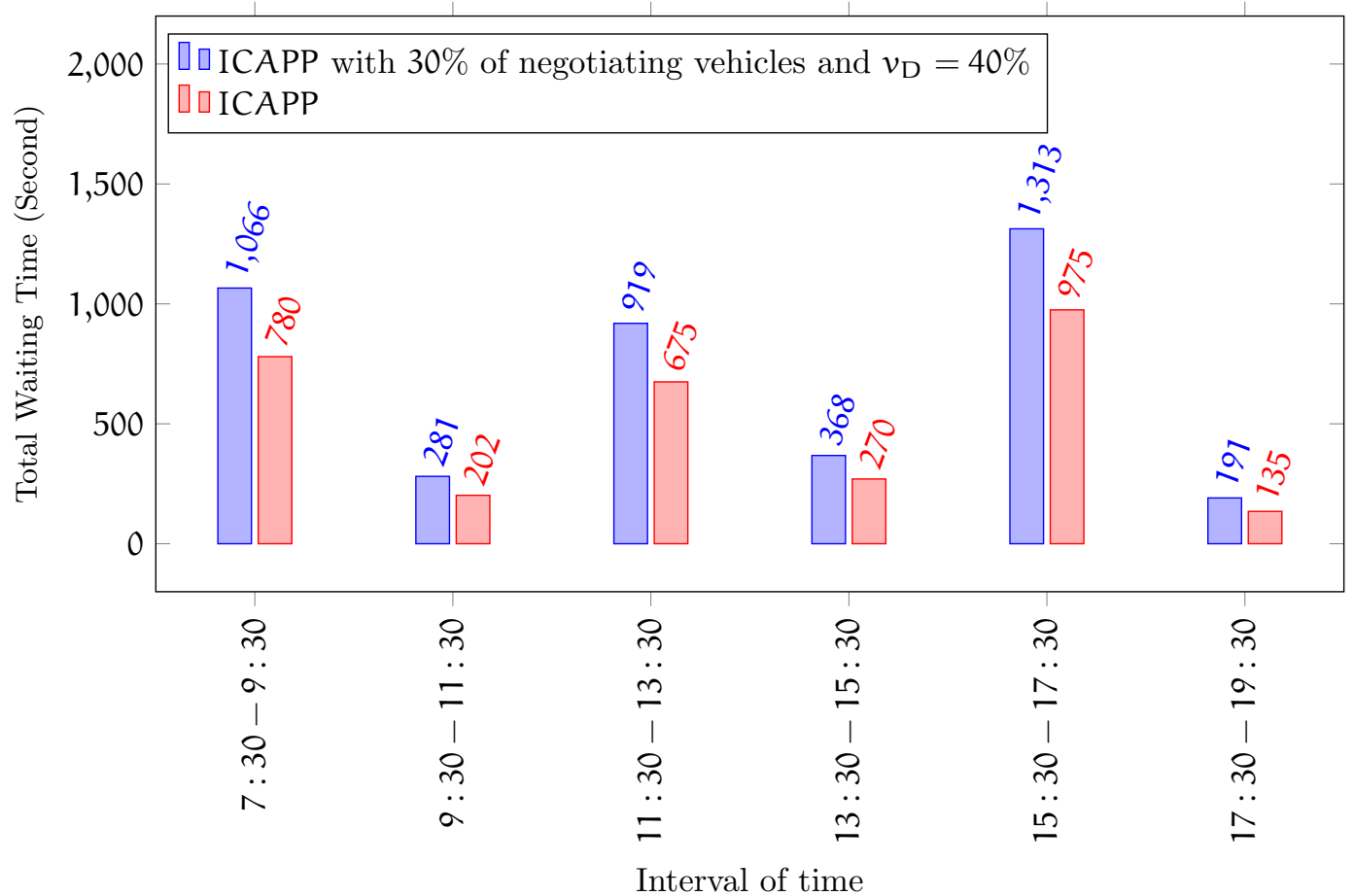

Figure 13: Total waiting time of priority vehicles with negotiating vehicles and $\nu_{\mathrm{D}}=40 \%$

\section{H) Impact of the proposed protocols on the environment}

In this section, we analyze the direct impact of the proposed protocols over the pollution indicator $\mathrm{CO}_{2}$. Table 5 presents the various factors, particularly related to the negotiating vehicles, which can affect their emissions of $\mathrm{CO}_{2}$. For example, the older and heavy vehicles consume more fuel, according to their acceleration/deceleration constantly to reach the recommended speed, compared to light-duty and new vehicles. Moreover, the fuel consumption in essence category of vehicles increases with their speed and decreases emissions of $\mathrm{CO}_{2}$. However, the diesel category consumes more fuel, and also produces more than $\mathrm{CO}_{2}$. In addition, another factor to consider here is the volume of dishonest vehicles.

As illustrated in Figure 14, we show the evolution graphs of $\mathrm{CO}_{2}$. The proposed protocols achieve significant improvements in pollutant emissions of $\mathrm{CO}_{2}$ in the range $43 \%-70 \%$, compared to circular case. The reference case ICANP with $V^{D}=0 \%$ significantly, reduces by $47 \%$ the emissions of $\mathrm{CO}_{2}$ compared to ICANP with $\mathrm{V}^{\mathrm{D}}=40 \%$, which proves that the presence of dishonest vehicles at signalized intersection increase the emissions of $\mathrm{CO}_{2}$. But, when we introduce the NPBR, the emissions of $\mathrm{CO}_{2}$ are decreased to $35 \%$ with an improvement of $12 \%$ compared to the reference case. 


\begin{tabular}{|c|c|c|c|c|}
\hline & & & Negotiating Vehicles Rate & Dishonest Vehicles Rate \\
\hline \multirow{4}{*}{ New vehicles } & \multirow{2}{*}{ Light-duty } & Essence & $35 \%$ & \multirow{8}{*}{$40 \%$} \\
\hline & & Diesel & $25 \%$ & \\
\hline & \multirow{2}{*}{ Heavy } & Essence & $15 \%$ & \\
\hline & & Diesel & $10 \%$ & \\
\hline \multirow{4}{*}{ Old vehicles } & \multirow{2}{*}{ Light-duty } & Essence & $06 \%$ & \\
\hline & & Diesel & $04 \%$ & \\
\hline & \multirow{2}{*}{ Heavy } & Essence & $03 \%$ & \\
\hline & & Diesel & $02 \%$ & \\
\hline
\end{tabular}

Table 5: Factors affecting $\mathrm{CO}_{2}$ emission

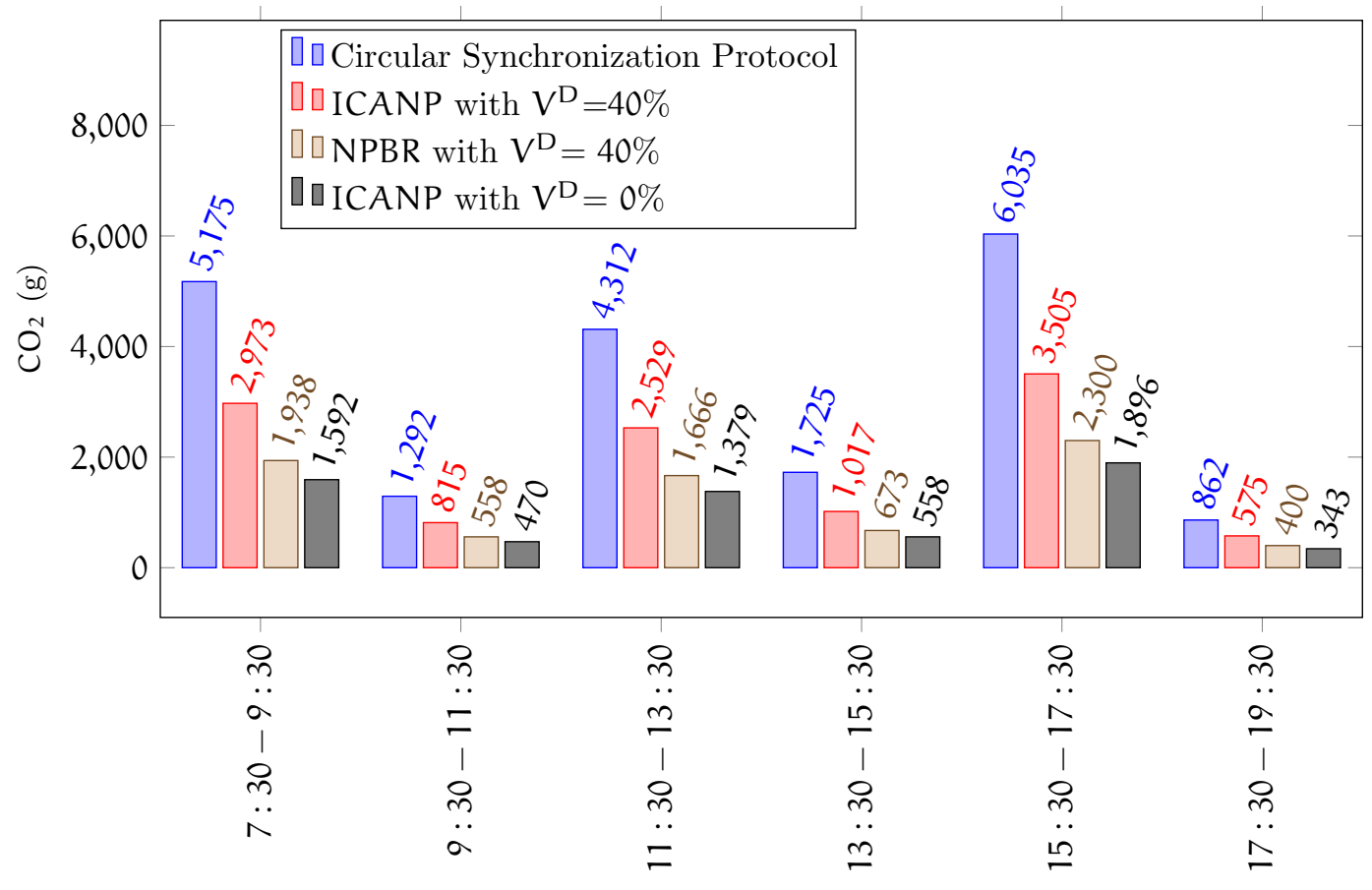

Interval of time

Figure 14: Emissions of $\mathrm{CO}_{2}$ in circular and negotiation cases, with and without dishonest vehicles

\subsection{Complexity Analysis}

To study the scalability of our proposal, we analyze the efficiency of our protocols in terms of the computation complexity. For this, we focus on the influence of number of vehicles circulating at the signalized intersection. The proposed protocols involve the optimization using GA. We analyze the computation cost of each one. In both protocols, GA is in charge of optimizing the crossing time of each vehicle in order to minimize its delay at the signalized intersection according to the presence or not of dishonest vehicles.

Table 6 shows the concrete analysis on computation operations and complexity of the proposed protocols. We note that the computation complexity of crossing time, effective distance and effective density function in GAICANP and volume of dishonest vehicle in GANBPR is in 
order of $\mathrm{O}(n)$. This means that the computation overhead of each $\mathrm{L}_{i}$ increases with the number $n$. However, the overhead of real distance and reputation computations are constant. We ignore the computation cost of these operations in our analysis. Also, the computation complexity of fitness function in GAICANP and GANBPR is in order of $\mathrm{O}\left(\mathrm{n}^{2}\right)$. This means that the computation overhead of each traffic light increases with $d_{i}^{e}$, and the number $n$ of traffic light in the system. However, it remains fixed with the effect of $V_{i}^{D}$. The computation complexity of both ICANP and NBPR is in order of $\mathrm{O}\left(\mathrm{n}^{2}\right)$ depending on the complexity of their fitness function. In order to analyze the complexity cost introduced by the proposed protocols, we define the time of each parameter transferred in the protocols. We set the time of both mathematical operations or comparison as $1 \mathrm{~ms}$. The affectation operation is set as 1,5 $\mathrm{ms}$, and the time of each reading, writing operation requires $0.5 \mathrm{~ms}$. We compared our protocols with ITLC 1 that are used in a similar application scenario presented in Table 6. ITLC considers the density of traffic flow to schedule the phases of each isolated traffic light at the signalized intersection. Hence, the computation complexity of ITLC is in order of $\mathrm{O}\left(\mathrm{n}^{3}\right)$, but both protocols are in order of $\mathrm{O}\left(\mathrm{n}^{2}\right)$.

\begin{tabular}{|c|c|c|c|c|}
\hline \multirow{2}{*}{ Protocol } & \multirow{2}{*}{ Phase } & \multirow{2}{*}{ Operations } & \multicolumn{2}{|c|}{ Complexity } \\
\hline & & & Partial & Global \\
\hline \multirow{7}{*}{ GAICANP } & Calculate crossing time & Equation 2 & $\mathrm{O}(\mathrm{n})$ & \multirow{7}{*}{$\mathrm{O}\left(\mathrm{n}^{2}\right)$} \\
\hline & Calculate real distance & Equation 4 & $\mathrm{O}(1)$ & \\
\hline & Calculate effective distance & Equation $\overline{5}$ & $\mathrm{O}(\mathrm{n})$ & \\
\hline & Effective Density Function & Algorithm 3 & $O(n)$ & \\
\hline & Fitness Function & Equation 6 & $\mathrm{O}\left(\mathrm{n}^{2}\right)$ & \\
\hline & - & Equation $\overline{7}$ & $\mathrm{O}(1)$ & \\
\hline & - & Equation 8 & $\mathrm{O}(1)$ & \\
\hline \multirow{3}{*}{ GANBPR } & Calculate Reputation & Equation 9 & $\mathrm{O}(1)$ & \multirow{3}{*}{$O\left(n^{2}\right)$} \\
\hline & Volume of dishonest vehicle & Algorithm 5 & $O(n)$ & \\
\hline & Fitness function & Equation 10 & $\mathrm{O}\left(\mathrm{n}^{2}\right)$ & \\
\hline \multirow{3}{*}{$\begin{array}{c}\text { ICANP } \\
\text { (Algorithm } 4)\end{array}$} & Case 1 & $2 \cdot n+2$ & $\mathrm{O}(\mathrm{n})$ & \multirow{3}{*}{$O\left(n^{2}\right)$} \\
\hline & Case 2 & $41 \cdot n+\frac{75}{2}$ & $\mathrm{O}\left(\mathrm{n}^{2}\right)$ & \\
\hline & Case 3 & $9 \cdot n^{2}+17 \cdot n+5$ & $O\left(n^{2}\right)$ & \\
\hline \multirow{5}{*}{$\begin{array}{c}\text { NBPR } \\
(\text { Algorithm 6) }\end{array}$} & Case 1 & $2 \cdot n+2$ & $\mathrm{O}(\mathrm{n})$ & \multirow{5}{*}{$\mathrm{O}\left(\mathrm{n}^{2}\right)$} \\
\hline & Case 2 & $103 \cdot n+\frac{99}{2}$ & $\mathrm{O}\left(\mathrm{n}^{2}\right)$ & \\
\hline & Passage verification & - & $\mathrm{O}(1)$ & \\
\hline & Decision & $9 \cdot n^{2}+17 \cdot n+5$ & $\mathrm{O}\left(\mathrm{n}^{2}\right)$ & \\
\hline & Post evaluation & Algorithm 7 & $\mathrm{O}(1)$ & \\
\hline ITLC [1] & & $2 \cdot n^{3}+2 \cdot n^{2}+\frac{41}{2} \cdot n$ & & $\mathrm{O}\left(\mathrm{n}^{3}\right)$ \\
\hline
\end{tabular}

Table 6: Computation complexity of the proposed protocols

Furthermore, we simulate the performances of the proposed protocols in terms of the computation. We tested the operation time of six main steps in our protocols. Since the effective density of negotiating vehicles affects the operation time of the different steps, we set $d_{i}^{e}$ from 10 to 160 with 20 as an increment. As shown in Figure 15, the operation time of crossing time computation (Equation 2), effective distance computation (Equation 5), effective density func- 
tion (Algorithm 3), and volume of dishonest vehicle (Algorithm 5 increases linearly with the number of negotiating vehicles at the signalized intersection. However, it is lightweight compared the fitness function with and without dishonest vehicles. Its cost increases linearly from the lowest execution time $395 \mathrm{~ms}$ to $5785 \mathrm{~ms}$ when the number of negotiating vehicles increases from 10 to 150. The reason is that only a few multiplication operations are performed in these steps compared to the fitness function, which consists of many pairing operations. Moreover, the fitness function in GANBPR (Equation 10 ) increases linearly with the number of negotiating vehicles greater than the fitness function in GAICANP (Equation 10). This fact could be due to the additional computing time of crossing time of dishonest vehicles.

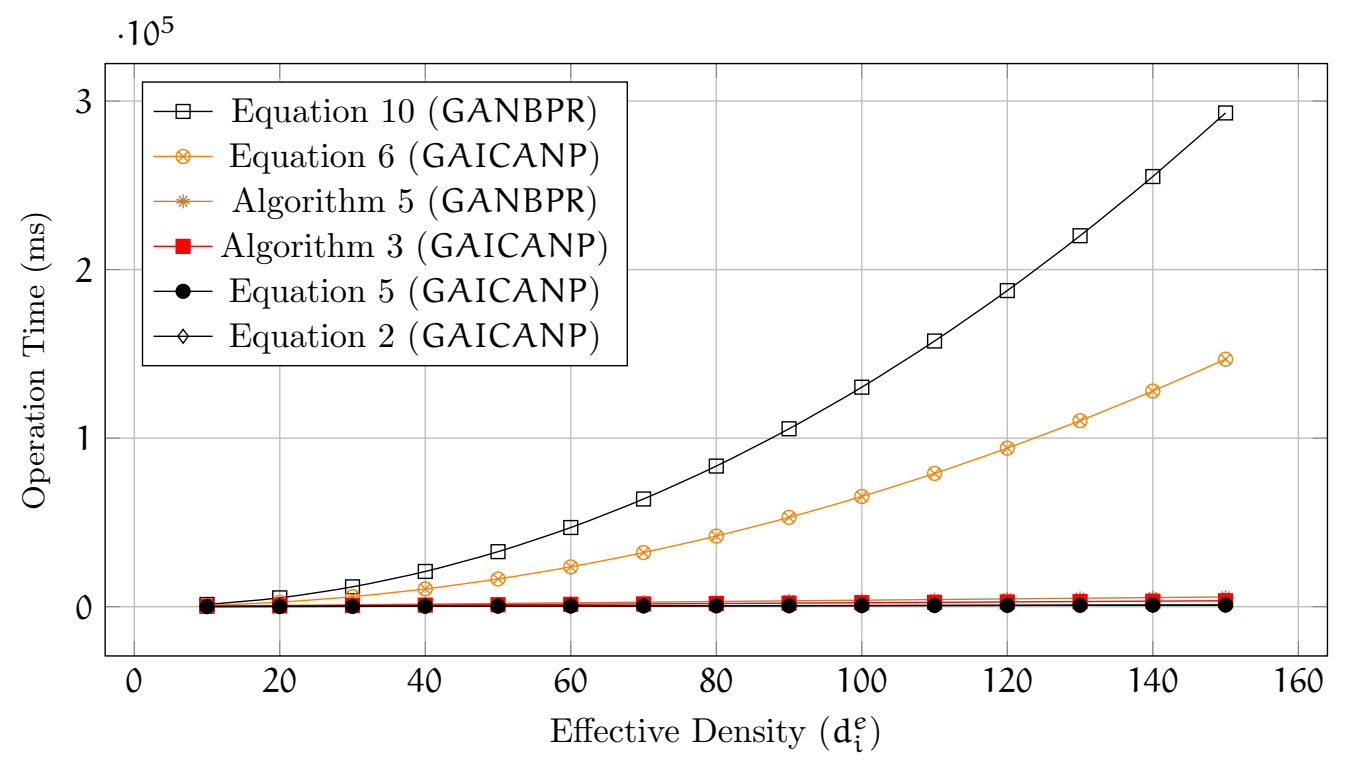

Figure 15: Complexity of optimization problem

We further compare the performances of the proposed protocols with ITLC [1] in Figure 16. The results demonstrate that the proposed protocols are more efficient than ITLC. According to the theoretical analysis, the operation time of the proposed protocols increases linearly with the number of negotiating vehicles. However, the operation time of ITLC increases with a complexity of $\mathrm{O}\left(\mathrm{n}^{3}\right)$.

\section{Conclusions}

In this paper, we propose context-aware negotiation, reputation, and priority traffic light scheduling protocols. The main objective is to reduce the congestion at the signalized intersections and to satisfy the drivers urgency by dynamically adapting the quantum of traffic lights. The protocol ICANP allows the negotiating vehicles to reach their destination according to their travel urgency. The effective density maximum of negotiating vehicles are scheduled to pass the road intersection first. On the other hand, it uses an optimization strategy based on GA that finds successful crossing time assigned at each phase. 


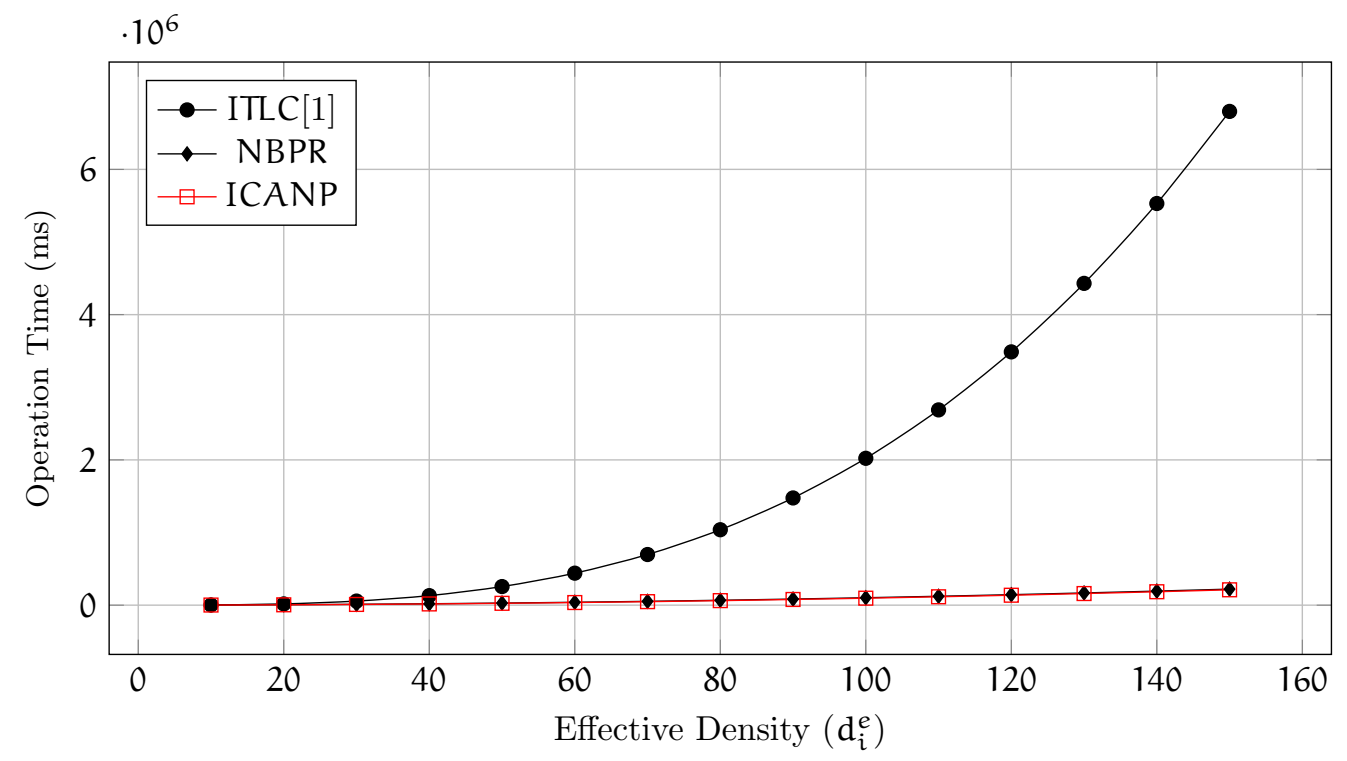

Figure 16: Comparison of operation time

The protocol NPBR allows each traffic light to check the reputation of its drivers. It introduces GA to reduce the effect of dishonest vehicles on the total waiting time of negotiating vehicles. The protocol ICAPP considers the existence of priority vehicles, while scheduling the phases of the traffic light cycle. Furthermore, ICAPP is designed for different scenarios considering the existence of other vehicles such as negotiating, dishonest and regular vehicles with priority. The obtained results are summarized as follows:

1. ICANP achieves significant improvements in terms of waiting time for negotiating vehicles. It decreases the total waiting time at each traffic light by $65 \%$ than the circular, and is $42 \%$ better than ITLC.

2. With the incoming dishonest vehicles at signalized intersections, NPBR reduces by $45 \%$ the waiting time for negotiating vehicles compared to ICANP, and by $64 \%$ compared to ITLC.

3. Where many priority vehicles reach the same signalized road intersection, ICAPP reduces by $32 \%$ the waiting time for priority vehicles compared to CATLS.

4. The priority vehicles density affects slightly the waiting time of the negotiating vehicles.

5. The negotiating and dishonest vehicles do not affect the waiting time of priority vehicles in conflicting traffic flow.

6. The proposed protocol achieves significant improvements following the pollution indicator $\mathrm{CO}_{2}$ with comparison to circular. ICANP with $\mathrm{V}^{\mathrm{D}}=0 \%$ reduces by $47 \%$ the $\mathrm{CO}_{2}$ emission compared to ICANP with $\mathrm{V}^{\mathrm{D}}=40 \%$. However, NPBR reduces by $35 \%$ the $\mathrm{CO}_{2}$ emission.

7. Formal complexity analysis and experimental tests show the efficiency and effectiveness of the proposed protocol compared to ITLC.

With regard to our future works, we plan to extend the study of large scale cities under different types of network topology and traffic density. Moreover, we are going to study other optimization techniques as well as other hybrid meta-heuristic to solve the congestion problem. 
For instance, PSO (Particle Swarm Optimization) and NSGA (Non-dominated Sorting Genetic Algorithm) could be good alternative solutions to improve the reliability of the problem resolution. Finally, we will examine the integrity of L2LCR messages between traffic lights in isolated and neighboring intersections.

\section{References}

[1] M. Bani-Younes and A. Boukerche. Intelligent Traffic Light Controlling Algorithms Using Vehicular Networks. IEEE Transaction on Vehicular Technology, Volume 65, Number 8, Pages 5887-5899, 2016.

[2] M. Bani-Younes, A. Boukerche, and A. Mammeri. Context-Aware Traffic Light SelfScheduling Algorithm for Intelligent Transportation Systems. IEEE Wireless Communications and Networking Conference, Pages 1-6, 2016.

[3] K. Pandit, D. Ghosal, H-M. Zhang, and C-H. Chuah. Adaptive Traffic Signal Control With Vehicular Ad hoc Networks. IEEE Transaction on Vehicular Technology, Volume 62, Number 4, Pages 1459-1471, 2013.

[4] C-T. Barba, M-A. Mateos, P-R. Soto, A-M. Mezher, and M-A. Igartua. Smart City For VANETs Using Warning Messages, Traffic Statistics and Intelligent traffic lights. IEEE Intelligent Vehicles Symposium, Pages 902-907, 2012.

[5] D. Krajzewicz, E. Brockfeld, J. Mikat, J. Ringel, C. Feld, W. Tuchscheerer, P. Wagner, and R. WÃ $\llbracket$ sler. Simulation of Modern Traffic Lights Control Systems Using the Open Source Traffic Simulation SUMO. In Proceedings of the $3^{\text {rd }}$ Industrial Simulation Conference, Pages 299-302, Berlin, 2005.

[6] C. Priemer and B. Friedrich. A Decentralized Adaptive Traffic Signal Control Using V2I Communication Data. In Proceedings of $12^{\text {th }}$ International IEEE Conference on Intelligent Transportation Systems, Pages 1-6, USA, 2009.

[7] J. Garcia-Nieto, A-C. Olivera, and E. Alba. Optimal Cycle Program of Traffic Lights With Particle Swarm Optimization. IEEE Trans. on Evolutionary Computation, Volume 17, Number 6, Pages 823-839, 2013.

[8] J. Garcia-Nieto, J. Ferrer, and E. Alba. Optimisation Traffic Lights with Metaheuristics: Reduction of Car Emissions and Consumption. In International Joint Conference on Neural Networks, Pages 48-54, China, 2016.

[9] Y-K. Chin, K-C. Yong, N. Bolong, S-S. Yang, and K-T-K. Teo. Multiple Intersections Traffic Signal Timing Optimization with Genetic Algorithm. IEEE International Conference on Control and Engineering, Pages 454-459, 2011.

[10] K-T-K. Teo, W-Y. Kow, and Y-K. Chin. Optimization of Traffic Flow within an Urban Traffic Light Intersection with Genetic Algorithm. Second International Conference on Computational Intelligence, Modeling and Simulation, Pages 172-177, 2010.

[11] L. Singh, S. Tripathi, and H. Arora. Time Optimization for Traffic Signal Control Using Genetic Algorithm. In International Journal of Recent Trends in Engineering, Volume 2, Number 2, 2009.

[12] Y. Li, L. Yu, S. Tao and K. Chen. Multi-Objective Optimization of Traffic Signal Timing for Oversaturated Intersection. Mathematical Problems in Engineering, Volume 2013, Number 8, Pages 9, 2013. 
[13] k. Deb, A. Pratap, S. Agarwal and T. Meyarivan . A fast and elitist multi objective genetic algorithm: NSGA-II. IEEE Transactions on Evolutionary Computation, Volume 6, Number 2, Pages 182-197, 2002.

[14] P. Gabriela, V. Naranjo, Z. Pooranian, M. Shojafar, M. Conti and R. Buyya. FOCAN: A. Fog-supported Smart City Network Architecture for Management of Applications in the Internet of Everything Environments. Journal of CoRR, Volume abs/1710.01801, 2017.

[15] F. Soylemezgiller, M. Kuscu, and D. Kilin. A Traffic Congestion Avoidance Algorithm with Dynamic Road Pricing for Smart Cities. In proceedings of Annual International Symposium on Personal, Indoor, and Mobile Radio Communications, Pages 2571-2575, 2013.

[16] O. Tomescu, I-M. Moise, A-E. Stanciu, and I. Batros. Adaptive Traffic Light Control System using Ad-hoc Vehicular Communications Network. Taiwanese Assoc. Artif. Intell., Volume 74, Number 2, Pages 1-8, 2012.

[17] M. Fogue, P. Garrido, F-J. Martinez, J. Cano, C-T. Calafate, P. Manzoni, and M. Sanchez. Prototyping an Automatic Notification Scheme for Traffic Accidents in Vehicular Networks. In proceedings of Wireless Days, Pages 1-5, 2011.

[18] S. Ganesh, A. Khekare, and V. Sakhare. A Smart City Framework for Intelligent Traffic System Using VANET. International Multi Conference on Automation, Computing, Communication, Control and Compressed Sensing, Pages 302-305, 2013.

[19] C. Dobre. Using Intelligent Traffic Lights to Reduce Vehicle Emissions. International Journal of Innovative Computing, Information and Control, Volume 8, Number 9, Pages 6283$6302,2012$.

[20] V. Gupta, R. Kumar, K-S. Reddy, and B-K. Panigrahi. Intelligent Traffic Light Control for Congestion Management for Smart City Development. Proceedings of the 10 Symposium, Pages 1-5, 2017.

[21] A-F. Santamaria and C. Sottile. Smart Traffic Management Protocol Based on VANET Architecture. Advances in Electrical and Electronic Engineering, Volume 12, Number 4, Pages 279-288, 2014.

[22] Y. Bravo, J. Luque, and E. Alba. Smart Mobility by Optimizing the Traffic Lights: A New Tool for Traffic Control Centers. First International Conference Smart-CT, Pages 147-156, Spain, 2016.

[23] W. Wen. A Dynamic and Automatic Traffic Light Control Expert System for solving the Road Congestion Problem. Expert Systems with Applications, Volume 34, Number 4, 2008.

[24] T. Khalid, A-N. Khan, M. Ali, A. Adeel, A-R. Khan, and J. Shuja. A fog-based security framework for intelligent traffic light control system. Multimedia Tools and Applications, DOI: https://doi.org/10.1007/s11042-018-7008-z. 